\title{
LISTA FLORISTICA COMENTADA DE PLANTAS VASCULARES SILVESTRES EN SAN JUAN QUETZALCOAPAN, TLAXCALA, MEXICO
}

\author{
HeIKE VibRans \\ Escuela de Ciencias \\ Universidad Autónoma del Estado de México \\ Instituto Literario 100 \\ Apartado postal 519 \\ 50000 Toluca, Estado de México
}

\begin{abstract}
RESUMEN
Se presenta una lista de 15 especies de pteridofitas y 381 de espermatofitas silvestres que se encontraron entre 1981 y 1993 en alrededor de $9 \mathrm{~km}^{2}$ de superficie al norte del volcán La Malinche. El área de estudio se ubica en las tierras cultivadas y perturbadas de San Juan Quetzalcoapan, municipio de Tzompantepec (2500 m s.n.m.). El principal cultivo es el maíz de temporal. El listado contiene el nombre científico, el nombre común local, la distribución general de la especie, el tipo de hábitat que ocupa en el área de estudio, observaciones sobre frecuencia y usos, los números y fechas de colecta, así como otros comentarios. Con este trabajo se pretende contribuir al conocimiento de la flora de un estado que hasta ahora es poco conocida. Además se muestra que aun ambientes muy alterados de México pueden poseer una diversidad biológica considerable.
\end{abstract}

\begin{abstract}
A checklist of 15 species of pteridophytes as well as of 381 spermatophytes is presented. The wildgrowing plants were collected between 1981 and 1993 for a study of the flora and vegetation of an area of about $9 \mathrm{~km}^{2}$ on the northern side of La Malinche volcano. The study area consists of the mestizo village of San Juan Quetzalcoapan, municipio of Tzompantepec, and its surroundings (2500 $\mathrm{m}$ above sea level). The main occupation is agriculture based on rain-fed maize cultivation. The checklist includes the following information: scientific name, name used locally, general distribution of the species, its habitat in the study area, observations on its frequency and uses, collection numbers and dates as well as commentaries. This report contributes to the inventory of the flora of a federal state not very well known up to now. It also shows that even the vegetation of very disturbed areas in Mexico can possess a considerable biological diversity.
\end{abstract}

\section{INTRODUCCION}

Tlaxcala, el estado más pequeño de la República Mexicana, no se conoce bien florísticamente, a pesar de su ubicación en el centro del país y de su accesibilidad. No ha atraído a los botánicos profesionales por ser un área eminentemente agrícola con poca vegetación primaria. 
En los últimos años, los botánicos del Jardín Botánico Tizatlán, cercano a la ciudad de Tlaxcala, han empezado una recolección sistemática en el estado y han publicado un listado provisional, enumerando 733 especies, de aproximadamente 2000 que se estiman para Tlaxcala (Acosta et al., 1991). Además, un grupo de investigadores de la misma especialidad de la Facultad de Estudios Superiores (FES-) Zaragoza, Universidad Nacional Autónoma de México, está trabajando en dicho estado.

Se considera útil dar a conocer un inventario de casi 400 especies (15 pteridofitas y 381 fanerógamas), con comentarios sobre nombres locales, usos, distribución y ecología. Tal enumeración es el resultado del estudio de la flora y vegetación de los terrenos de un pueblo agrícola ubicado en las faldas norte del volcán La Malinche (Apéndice 1). El listado provisional arriba mencionado no incluye 149 de las especies encontradas en San Juan.

En el apéndice 2 se enumeran las colectas y los herbarios de depósito de los ejemplares. En el apéndice 3 se ordenan alfabéticamente los nombres comunes, anexando sus nombres científicos correspondientes.

Es de enfatizar el hecho de que incluso la vegetación profundamente alterada de un ambiente afectado por la agricultura desde tiempos antiguos puede estar compuesta por un alto número de especies, la gran mayoría de ellas nativas. Esta considerable diversidad está amenazada en muchos lugares del centro de México por el éxito de algunas plantas foráneas (véanse los comentarios acerca del pasto Pennisetum clandestinum).

\section{METODOS}

La parte principal de la investigación se llevó a cabo en 1981 y 1982, habiéndose colectado aproximadamente 1100 especímenes. También se hicieron varias visitas entre 1988 y 1993, obteniendo otros 210 números.

El primer juego de ejemplares de herbario todavía lo conserva la autora, pero se depositará en el futuro herbario de la Facultad de Ciencias, Universidad Autónoma del Estado de México, El Cerrillo, Toluca. En el apéndice 2 se indican los ejemplares que fueron depositados en MEXU, ENCB, B o CHAP.

Los informantes de los nombres locales y los usos fueron principalmente los miembros de la familia de Don Frumencio Rodríguez, especialmente Doña Virginia Pérez de Rodríguez, aunque también se entrevistó a otros habitantes de San Juan de manera informal y no tan sistemática. Los datos se registraron durante el prensado de los especímenes en 1981. En 1982 se confirmaron las notas de todas las plantas colectadas con los mismos informantes por medio de un herbario de campo y de fotografías, y se corrigieron algunos detalles. Para los ejemplares obtenidos después de 1988 se tiene menos datos de esta índole.

Los nombres comunes mencionados en el apéndice 3 provienen todos de San Juan, ninguno se ha tomado de la literatura. Se procuró escribir los vocablos de origen náhuatl con el alfabeto español como se usa en México. Por ejemplo, se definió con una x el sonido que en inglés corresponde a sh. Si hay más de una manera de pronunciar un nombre, se enumeran todas las variantes (p.ej. jaramao y jaramado para Raphanus raphanistrum). Se adaptó la ortografía a la rutina de literatura especializada, pero sin sacrificar la usanza 
local. Por ejemplo, Plantago major generalmente se llama llantén en el centro de México, pero en San Juan lo denominan lantén.

La información detallada sobre el uso de las plantas, particularmente las medicinales (recetas, cantidades etc.) se publicará aparte. Igualmente se describirán la agricultura, la vegetación y los aspectos fitogeográficos en otra contribución. Sin embargo, el apéndice 1 ya contiene algunos datos básicos que atañen a estos temas, a los que se hará referencia posteriormente.

\section{EL AREA DE ESTUDIO}

San Juan Quetzalcoapan está ubicado en las faldas norte del volcán La Malinche; se encuentra sobre un pequeño cerro visible desde la carretera Apizaco-Huamantla, a $19^{\circ} 22^{\prime}$ N, $98^{\circ} 04^{\prime}$ W. Pertenece al municipio de San Salvador Tzompantepec. En 1981 tenía aproximadamente 1100 habitantes. Es una comunidad mestiza, pero establecida en un área poblada desde hace miles de años; en varios sitios se pueden encontrar navajas de obsidiana en abundancia.

La principal ocupación de la tierra es el cultivo de maíz criollo de temporal, con pequeñas superficies sembradas de avena (Avena), trigo (Triticum), cebada (Hordeum), frijol (Phaseolus vulgaris), ayocote (Phaseolus coccineus), haba (Vicia faba), chícharo (Pisum), alfalfa (Medicago) y flor de muerto (Tagetes). Debido al corredor industrial cercano y a las oportunidades de empleo que ofrecen Apizaco y otras ciudades del área, una proporción relativamente alta de habitantes se dedica a la agricultura sólo en forma parcial.

El pueblo está ubicado a unos 2500 m s.n.m. Recibe alrededor de $800 \mathrm{~mm}$ de precipitación anual, aunque con desviaciones grandes que en algunos años llegan a causar serios daños a la cosecha, sobre todo por la sequía. En los meses de enero y febrero hay heladas nocturnas diarias con niebla.

La vegetación natural del lugar corresponde al bosque de pino y encino, en el límite entre sus formas semihúmeda y semiárida (Klink, 1973). Los suelos son cambisoles de diferentes tipos y su textura varía de arenas limosas a limos arenosos; su pH (en agua $1: 2.5)$ se determinó en 11 sitios de campos de cultivo, obteniéndose valores entre 5.1 y 8.6, en su mayoría de alrededor de 6.

La superficie muestreada abarcó el cerro en el que se encuentra ubicado el centro del pueblo. Sus límites los constituyen: la Barranca de las Armas hacia el oeste y San Salvador, terrenos con fábricas hacia el norte, la carretera y San Cosme, el pie del cerro y unas barranquillas hacia el este y un bosque hacia el sur. El área de estudio cubre una extensión de aproximadamente $3 \mathrm{~km} \times 3 \mathrm{~km}$, o sea más o menos 900 hectáreas.

\section{LISTADO FLORISTICO}

En el apéndice 1 se proporciona en forma abreviada información sobre la distribución general de las especies, el tipo de vegetación en el que habitan principalmente, su frecuencia y su uso. La nomenclatura se rige en general por Rzedowski y Rzedowski (1979, 
1985, 1990); de estas obras también se tomaron la mayoría de los datos acerca de la distribución general de las especies. Se actualizaron algunos nombres y distribuciones con la Flora Novo-Galiciana (McVaugh, 1984, 1985, 1987) y con otros trabajos especializados. La clasificación de familias de los helechos es la que adoptó el comité de la Flora de Norteamérica en 1993.

En el apéndice 2 se citan los ejemplares colectados con número y fecha, indicando así la temporada de floración; además se señala el herbario donde se encuentra depositado un duplicado.

En el apéndice 3 se presentan los nombres comunes en secuencia alfabética y los nombres científicos correspondientes.

\section{AGRADECIMIENTOS}

La elaboración de este trabajo ha requerido de la intervención de muchas personas. Sin el apoyo, los conocimientos y la hospitalidad de la familia Rodríguez Pérez, de San Juan Quetzalcoapan, su realización no hubiera sido posible. La Biól. Ma. del Rocío Azcárraga Rosette me presentó con ellos. El Dr. W. Lauer (Bonn) sugirió el tema y asesoró mi tesis, en la cual se basa este artículo. En la Ciudad de México me ayudaron de diversas maneras, principalmente a través de la determinación de ejemplares y el apoyo con la literatura: Ma. de la Luz Arreguín, Robert Bye, Graciela Calderón de Rzedowski, Patricia Dávila, Francisco Espinosa, Judith Espinosa, Gabriel Flores, Raquel Galván, Abisaí García, Yolanda Herrera, Ernesto Jáuregui, Rafael Lira, David Lorence, José Luis Ornelas, Magdalena Peña, T. P. Ramamoorthy, Jerónimo Reyes, Lourdes Rico, Concepción Rodríguez, Gerardo Salazar, Mario Sousa y José Luis Villaseñor. Hildemar Scholz (Berlín), Klaus Kramer (antes Bonn) y J. A. Ratter (Edinburgo) me ayudaron con la determinación de algunas especies difíciles. Fernando Chiang, Luis Pinzón, Carlos Aguilar, Manuel Muñoz y un revisor anónimo leyeron todo o partes del manuscrito e hicieron numerosas correcciones y sugerencias. A todos ellos van mis más sinceros agradecimientos.

\section{LITERATURA CITADA}

Acosta Pérez, R., G. L. Galindo Flores y L. V. Hernández Cuevas. 1991. Listado preliminar de la flora fanerogámica del estado de Tlaxcala. Gobierno del Estado de Tlaxcala. Tlaxcala, Tlax. Folleto 12. pp. 4-44.

Fassett, N. C. 1951. Callitriche in the New World. Rhodora 53: 137-155, 161-182, 185-194, 209-222.

Flora of North America Editorial Committee. 1993. Flora of North America north of Mexico. Pteridophytes and Gymnosperms. Vol. 2. Oxford University Press, Nueva York. Oxford. 475 pp.

Klink, H. J. 1973. Die natürliche Vegetation und ihre räumliche Ordnung im Puebla-Tlaxcala Gebiet (Mexiko). Erdkunde 27: 213-225.

Martínez, M. 1979. Catálogo de nombres vulgares y científicos de plantas mexicanas. Fondo de Cultura Económica. México, D.F. 1247 pp.

McVaugh, R. 1984. Flora Novo-Galiciana. A descriptive account of the vascular plants of Western Mexico. Compositae. Vol. 12. University of Michigan. Ann Arbor. $1161 \mathrm{pp}$.

McVaugh, R. 1985. Flora Novo-Galiciana. A descriptive account of the vascular plants of Western Mexico. Orchidaceae. Vol. 16. University of Michigan. Ann Arbor. 363 pp. 
McVaugh, R. 1987. Flora Novo-Galiciana. A descriptive account of the vascular plants of Western Mexico. Leguminosae. Vol. 5. University of Michigan. Ann Arbor. 786 pp.

Rzedowski, J. y G. C. de Rzedowski. 1979. Flora fanerogámica del Valle de México. Vol. 1. Compañía Editorial Continental, S. A. México, D.F. 403 pp.

Rzedowski, J. y G. C. de Rzedowski. 1985. Flora fanerogámica del Valle de México. Vol. 2. Escuela Nacional de Ciencias Biológicas e Instituto de Ecología. México, D. F. 674 pp.

Rzedowski, J. y G. C. de Rzedowski. 1990. Flora fanerogámica del Valle de México. Vol. 3. Instituto de Ecología. Pátzcuaro, Mich. 494 pp. 


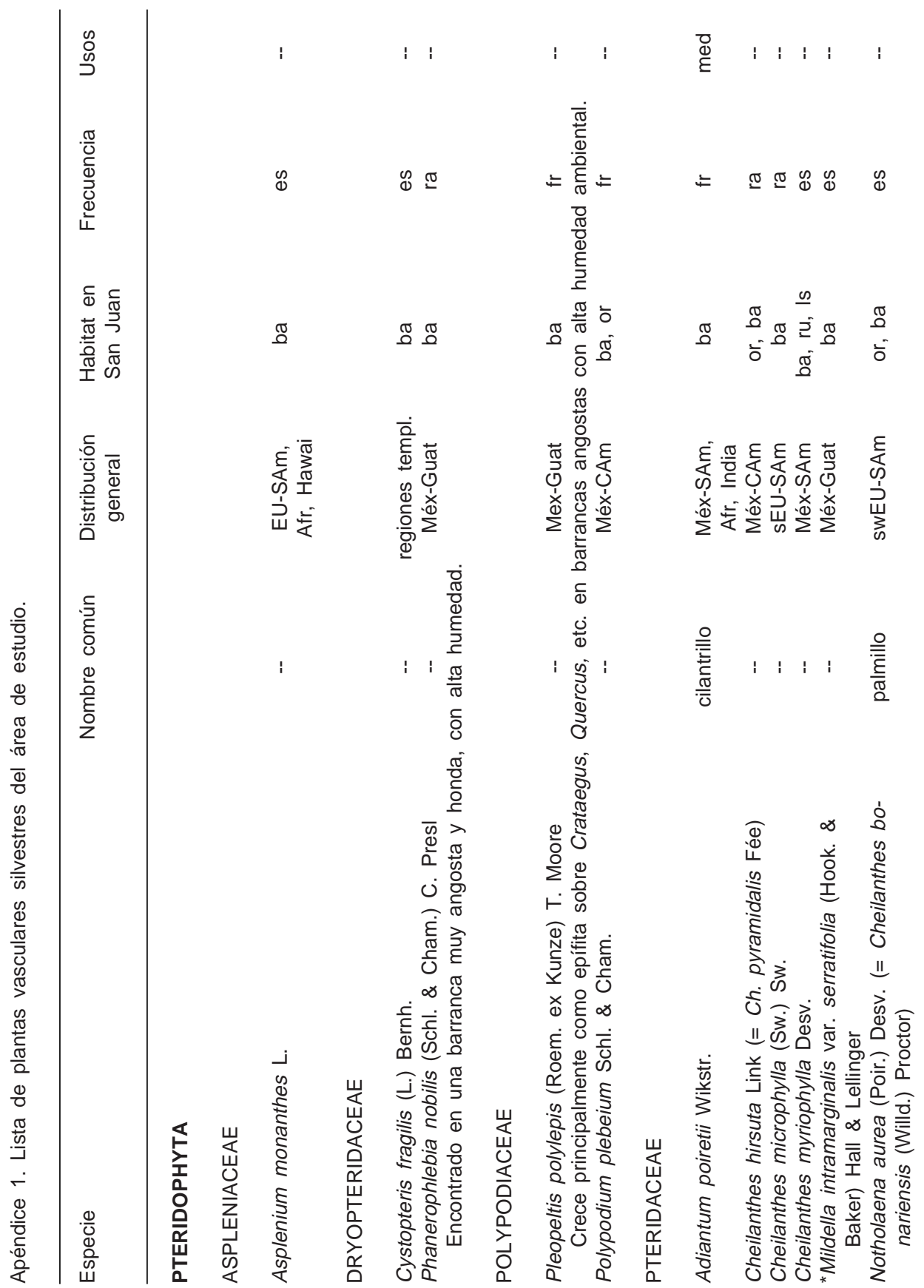


Vibrans: Plantas Vasculares Silvestres en San Juan Quetzalcoapan, Tlaxcala

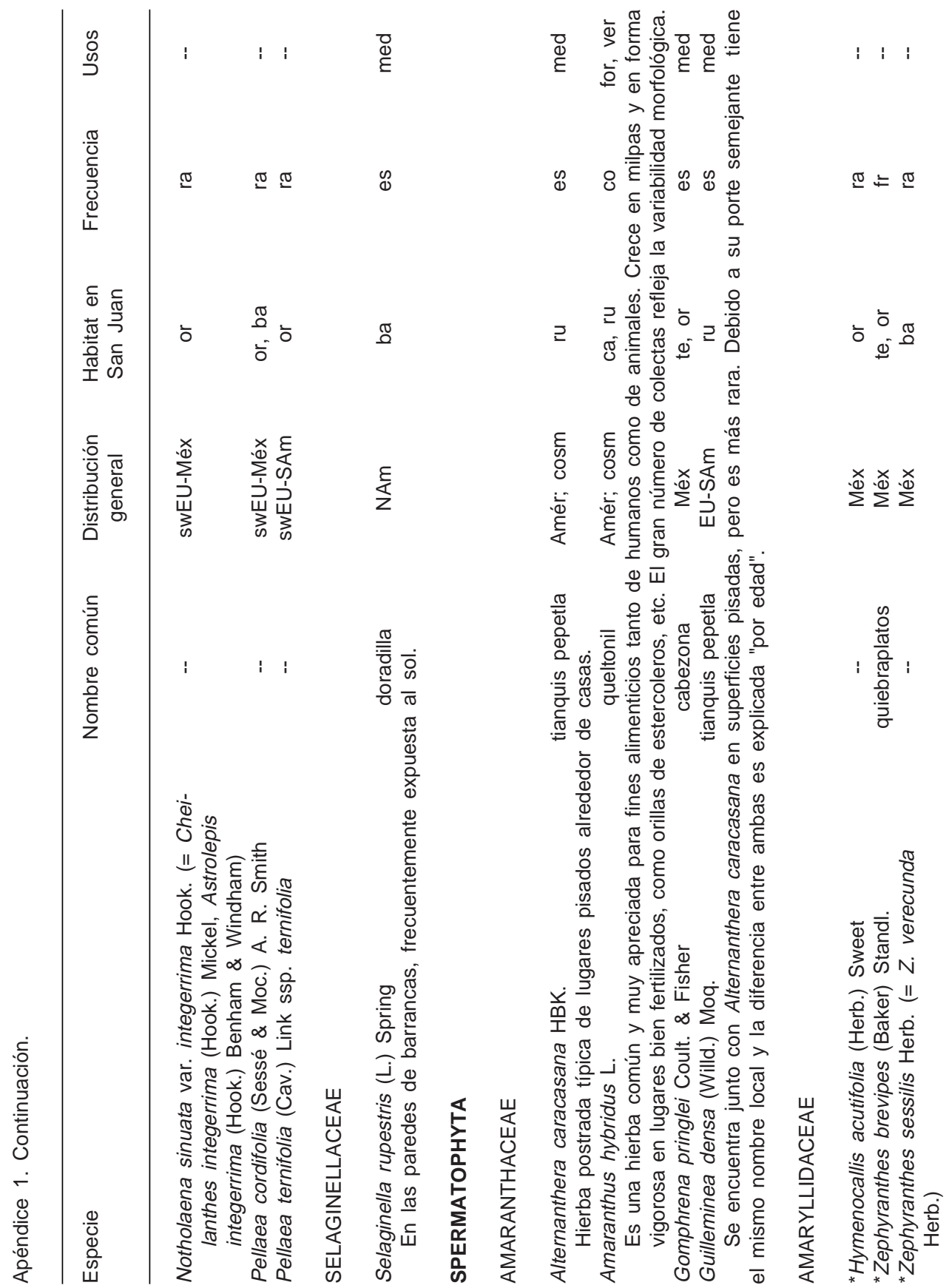




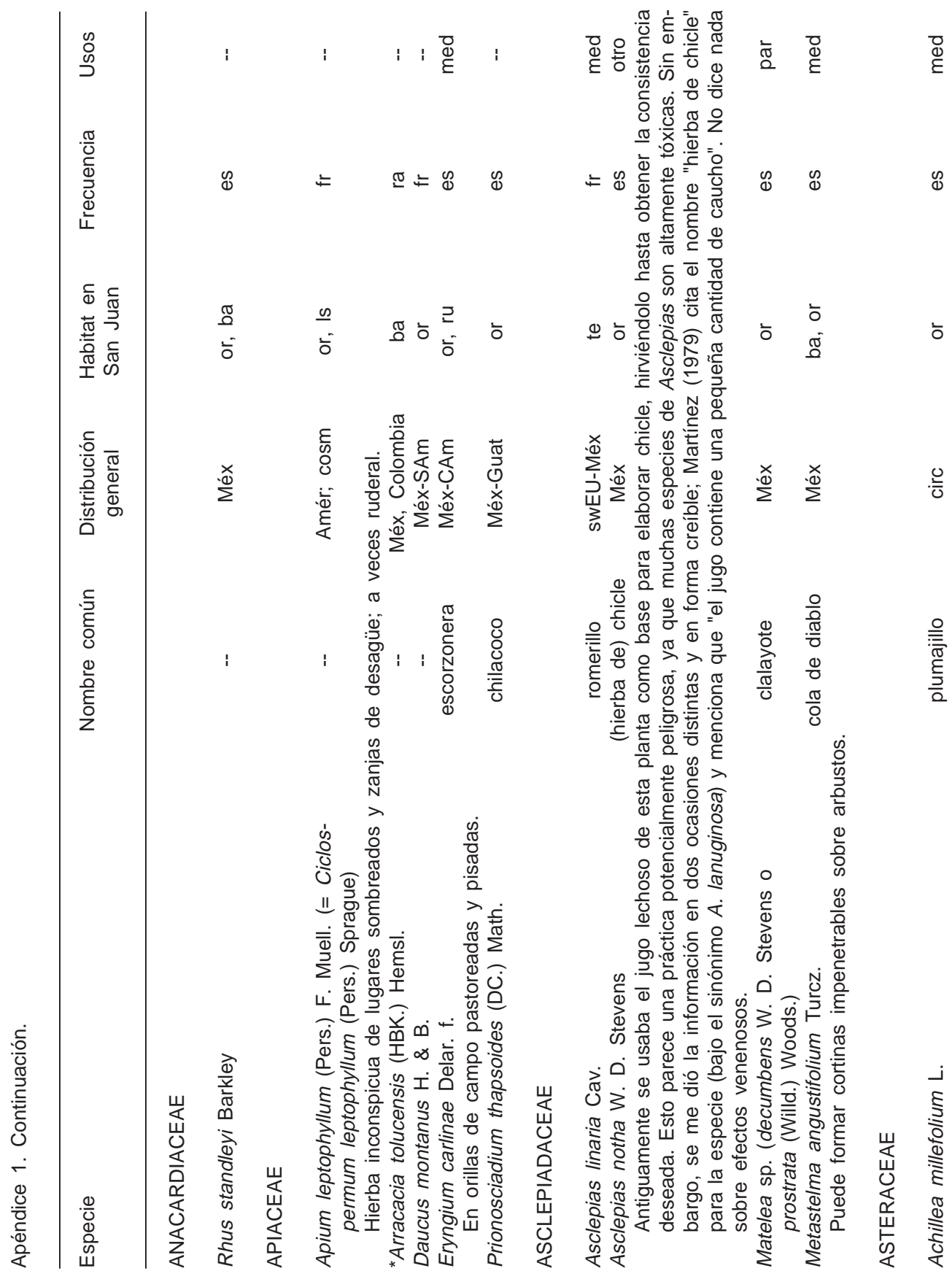


Vibrans: Plantas Vasculares Silvestres en San Juan Quetzalcoapan, Tlaxcala

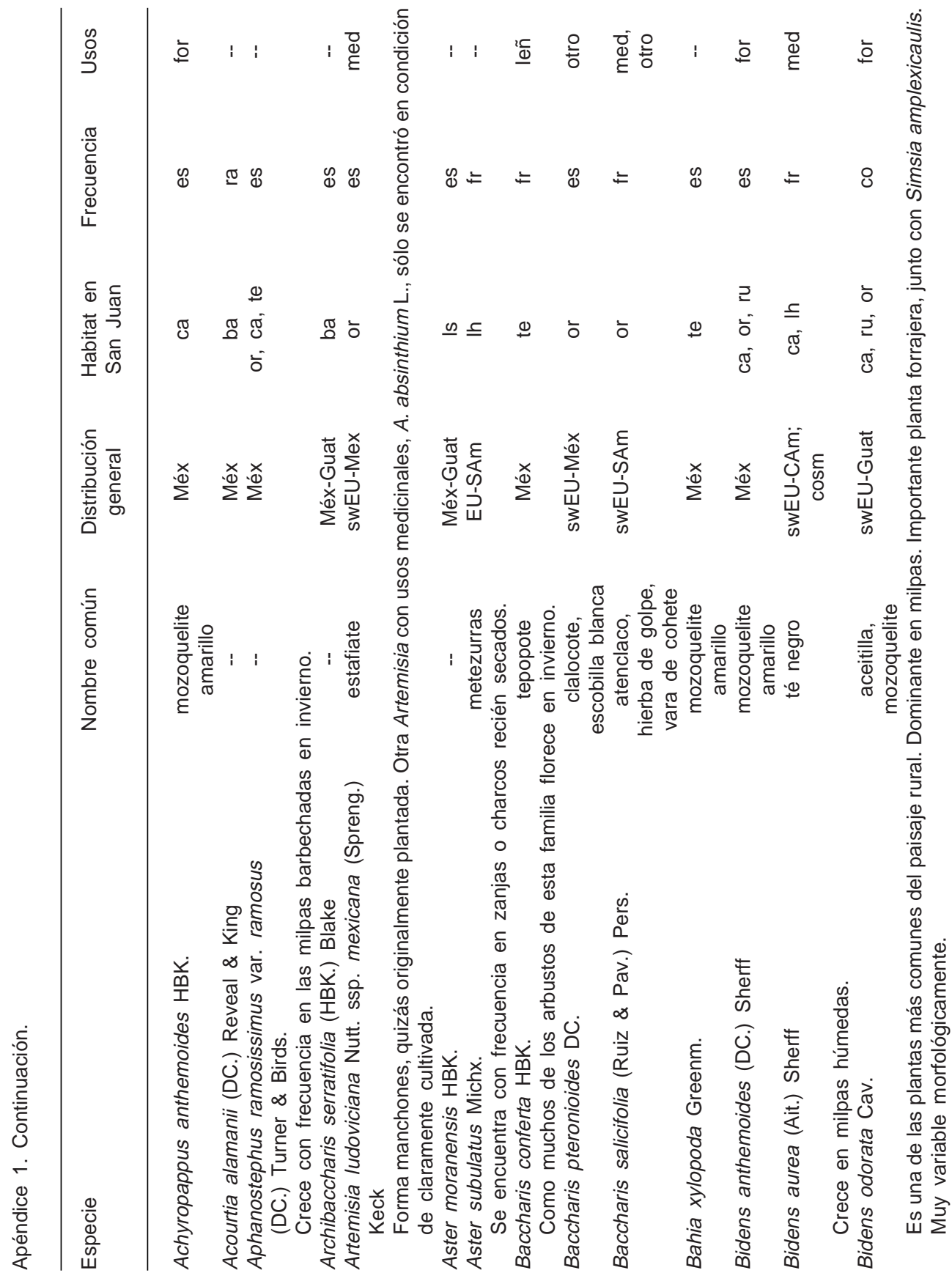




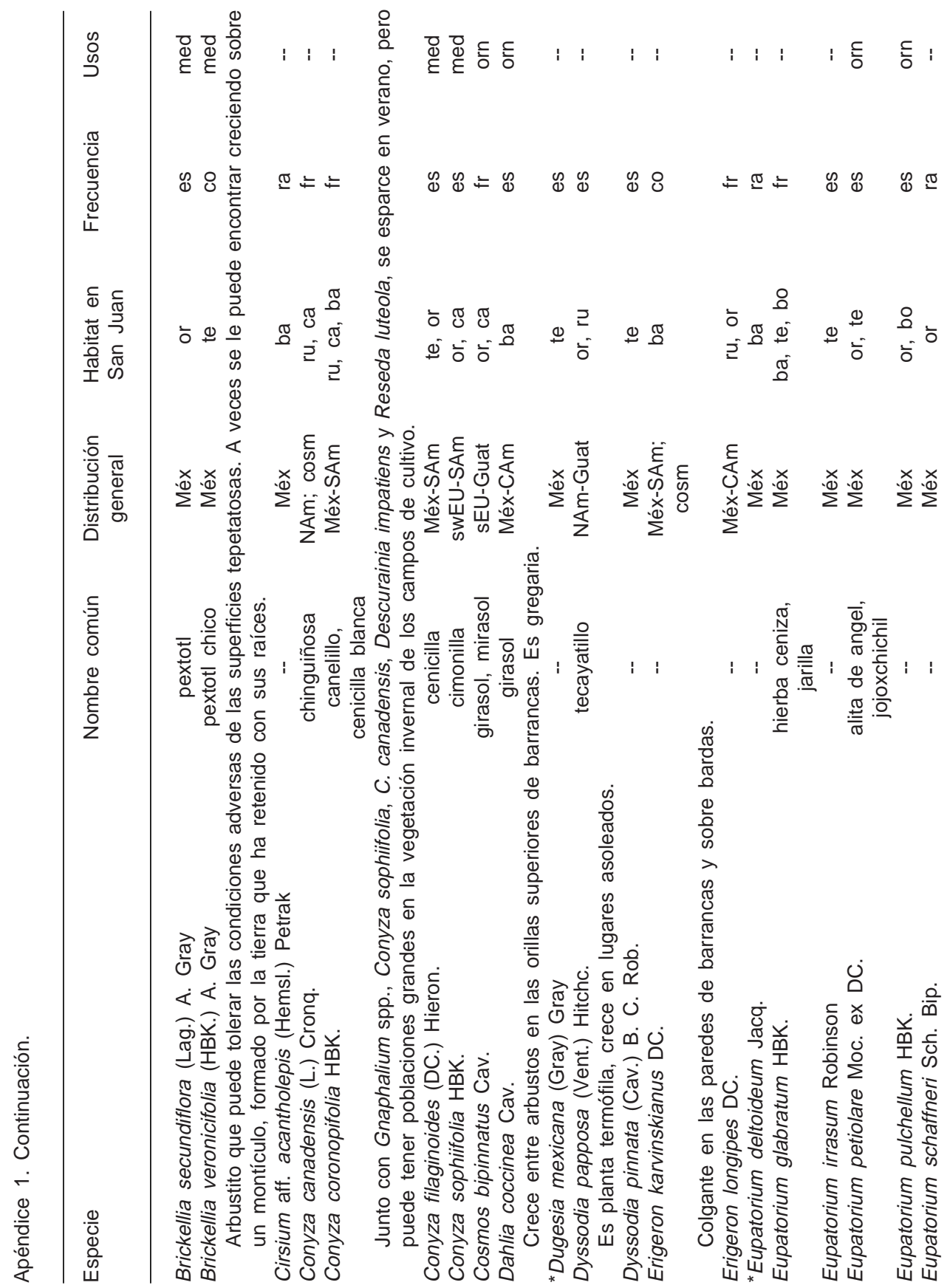


Vibrans: Plantas Vasculares Silvestres en San Juan Quetzalcoapan, Tlaxcala

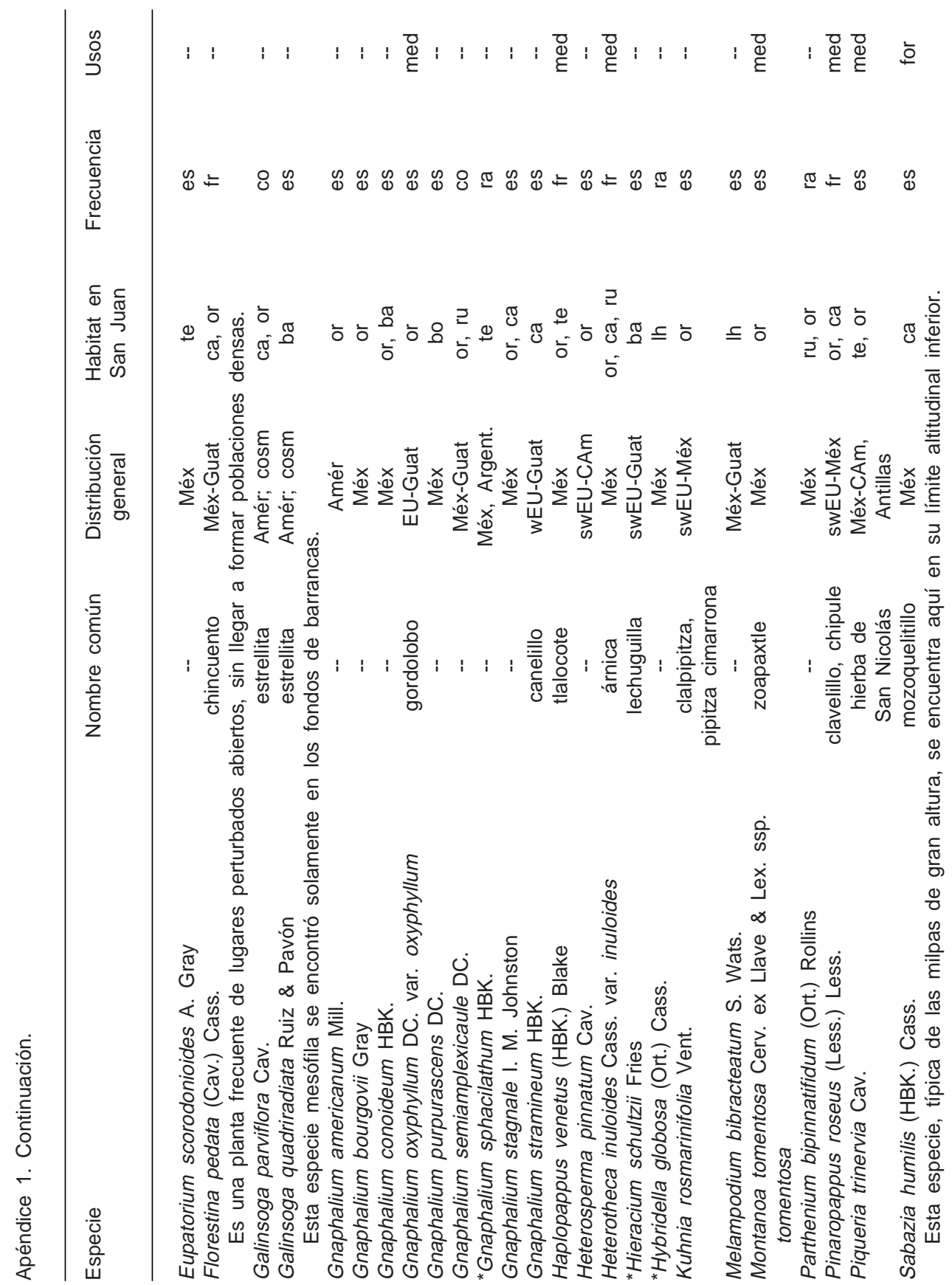




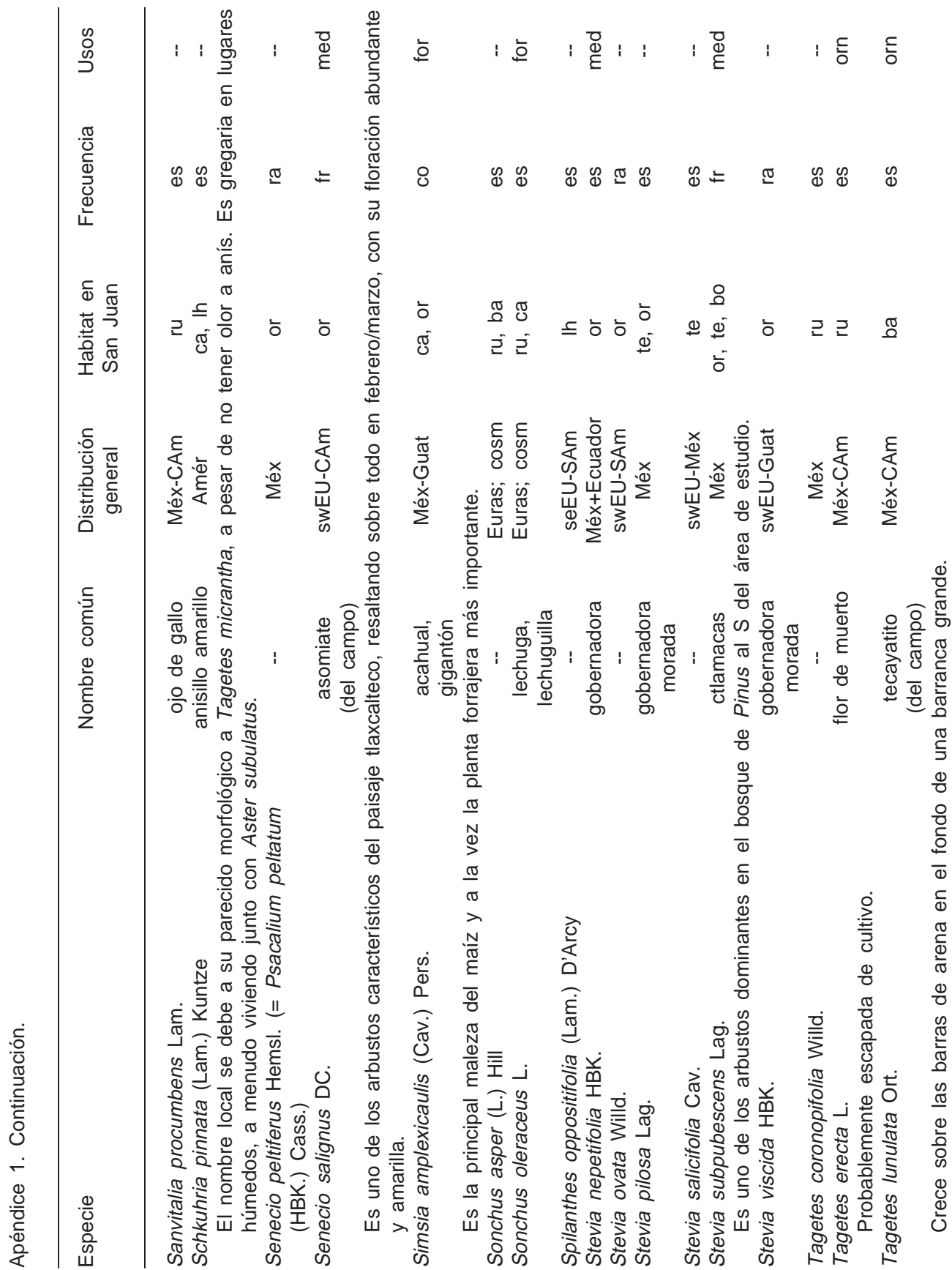


Vibrans: Plantas Vasculares Silvestres en San Juan Quetzalcoapan, Tlaxcala

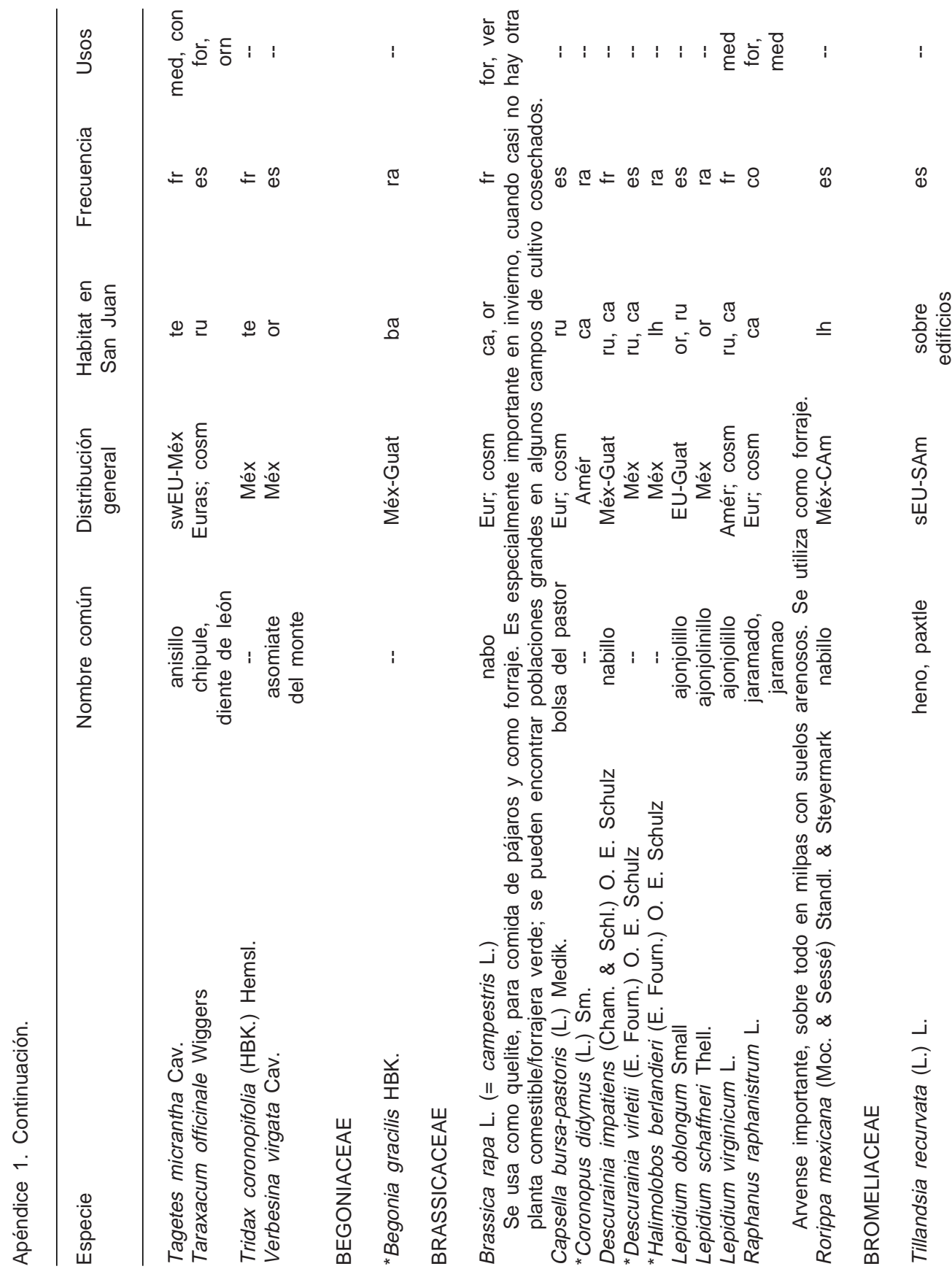




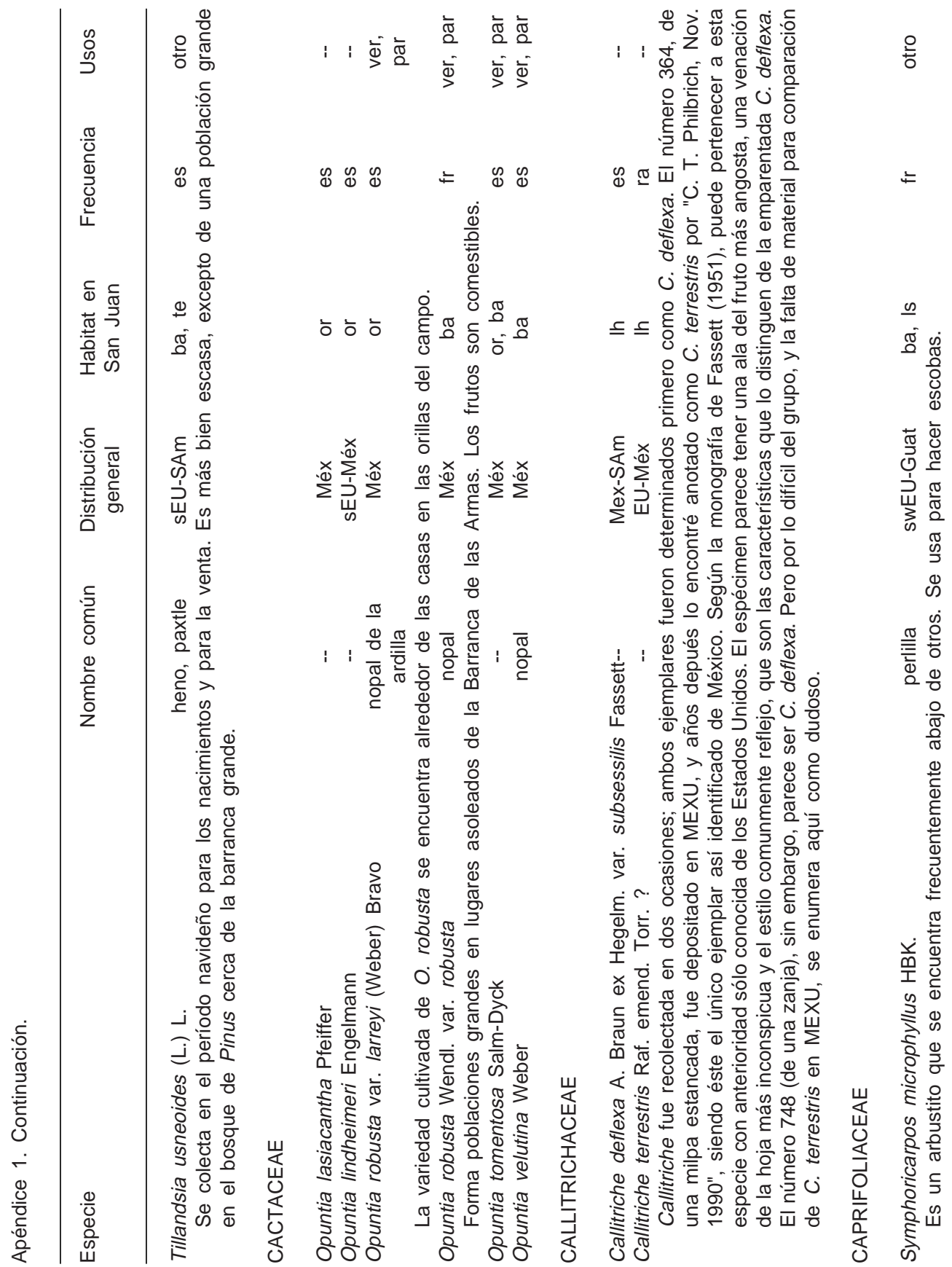


Vibrans: Plantas Vasculares Silvestres en San Juan Quetzalcoapan, Tlaxcala

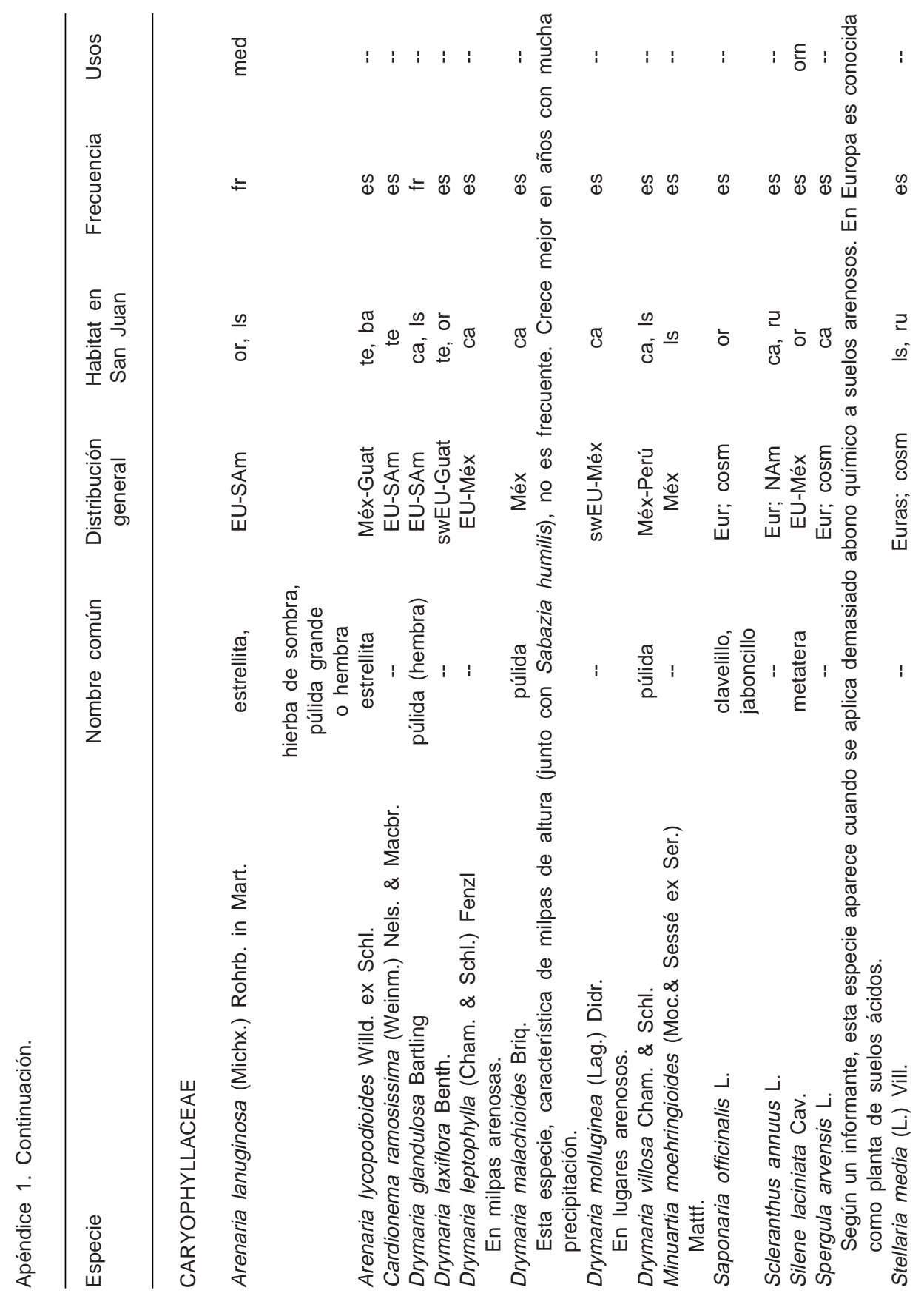




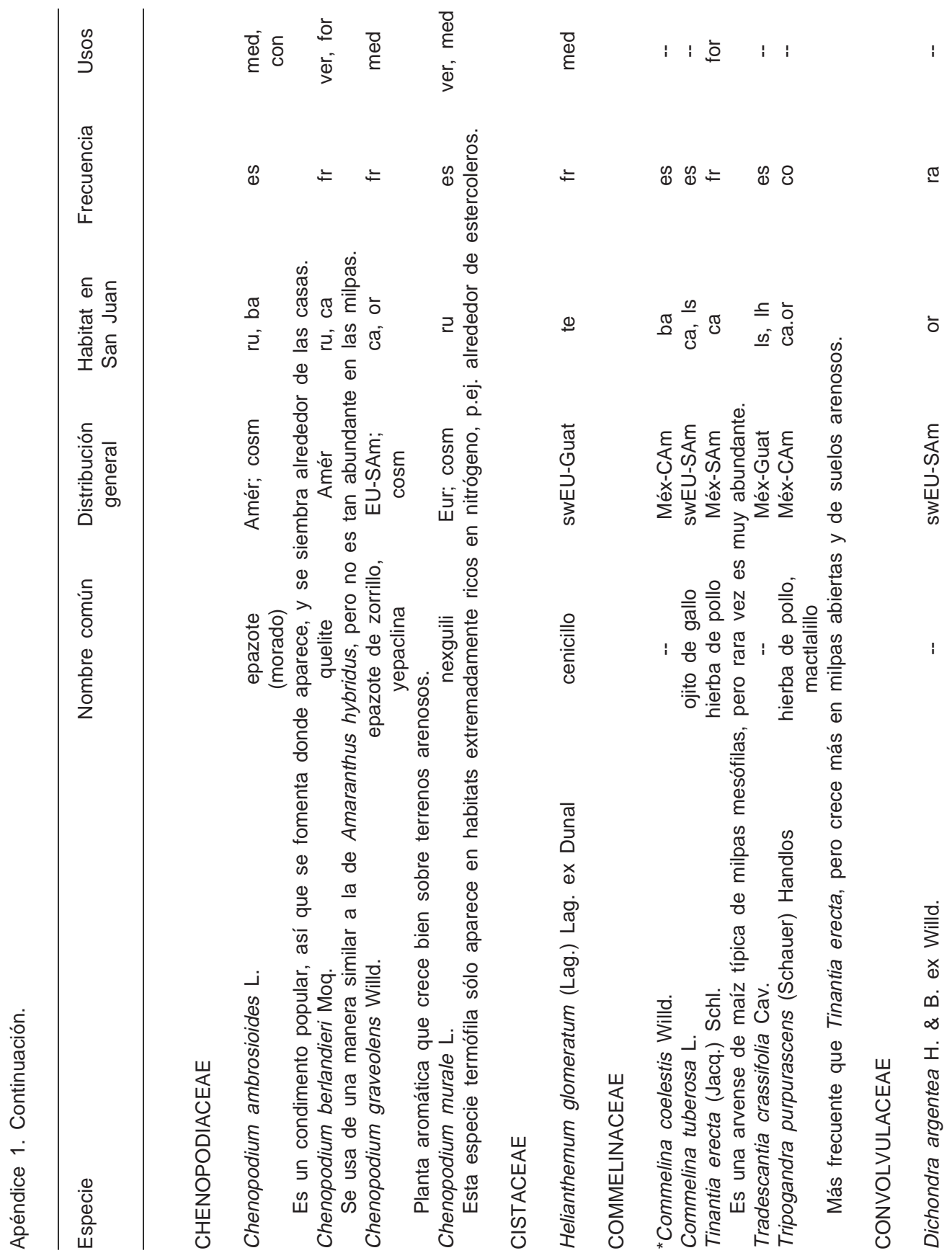


Vibrans: Plantas Vasculares Silvestres en San Juan Quetzalcoapan, Tlaxcala

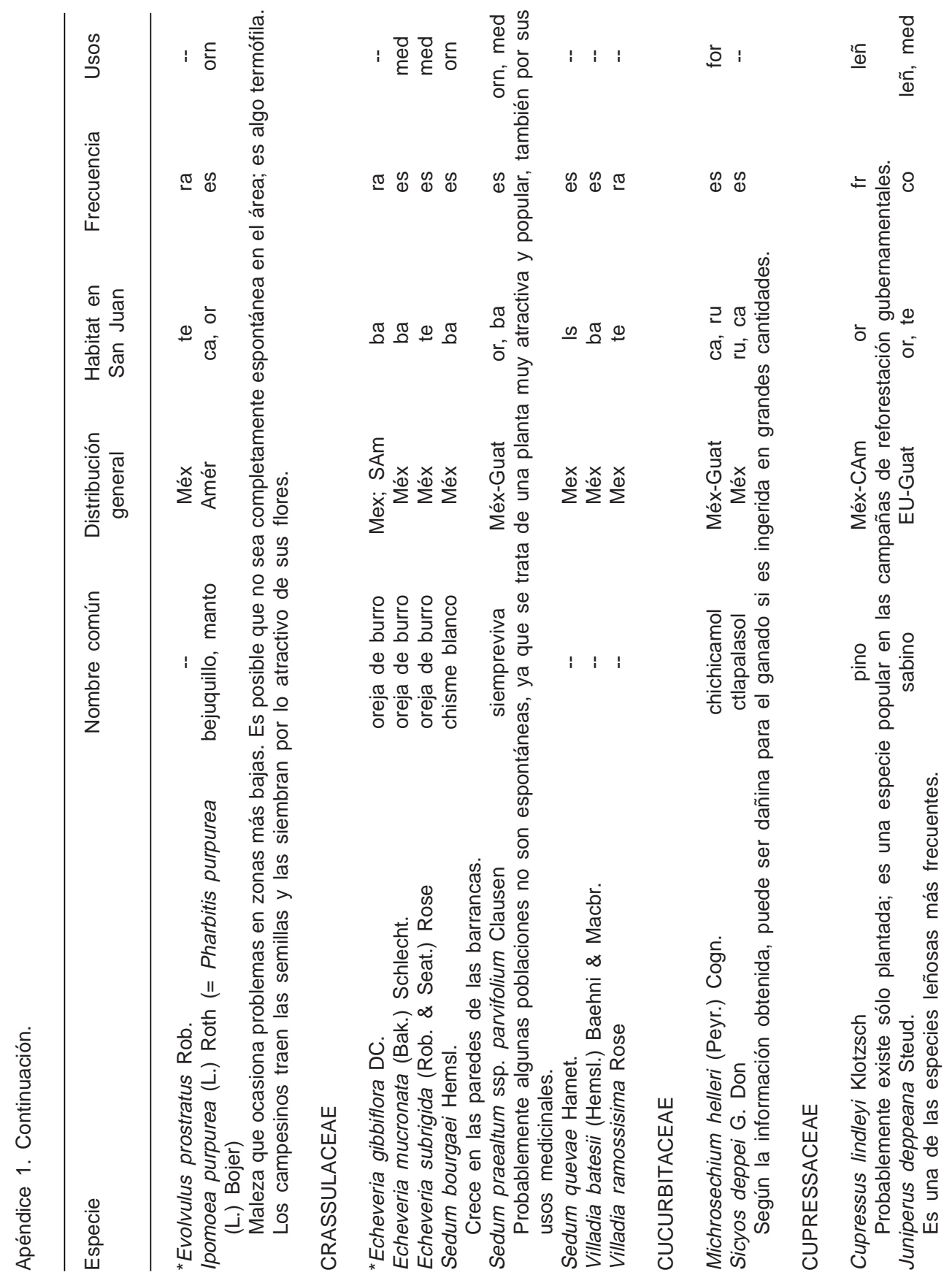




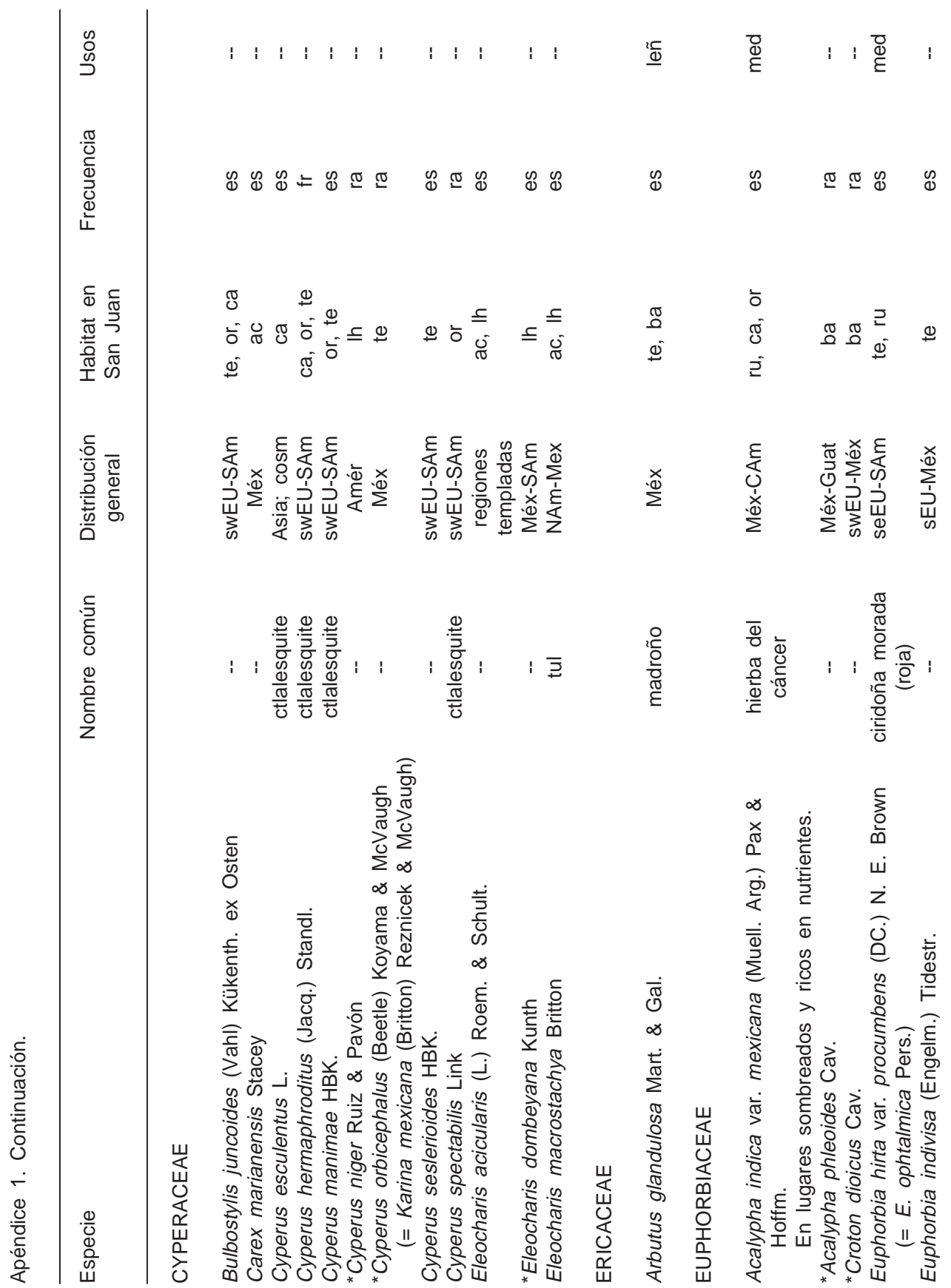


Vibrans: Plantas Vasculares Silvestres en San Juan Quetzalcoapan, Tlaxcala

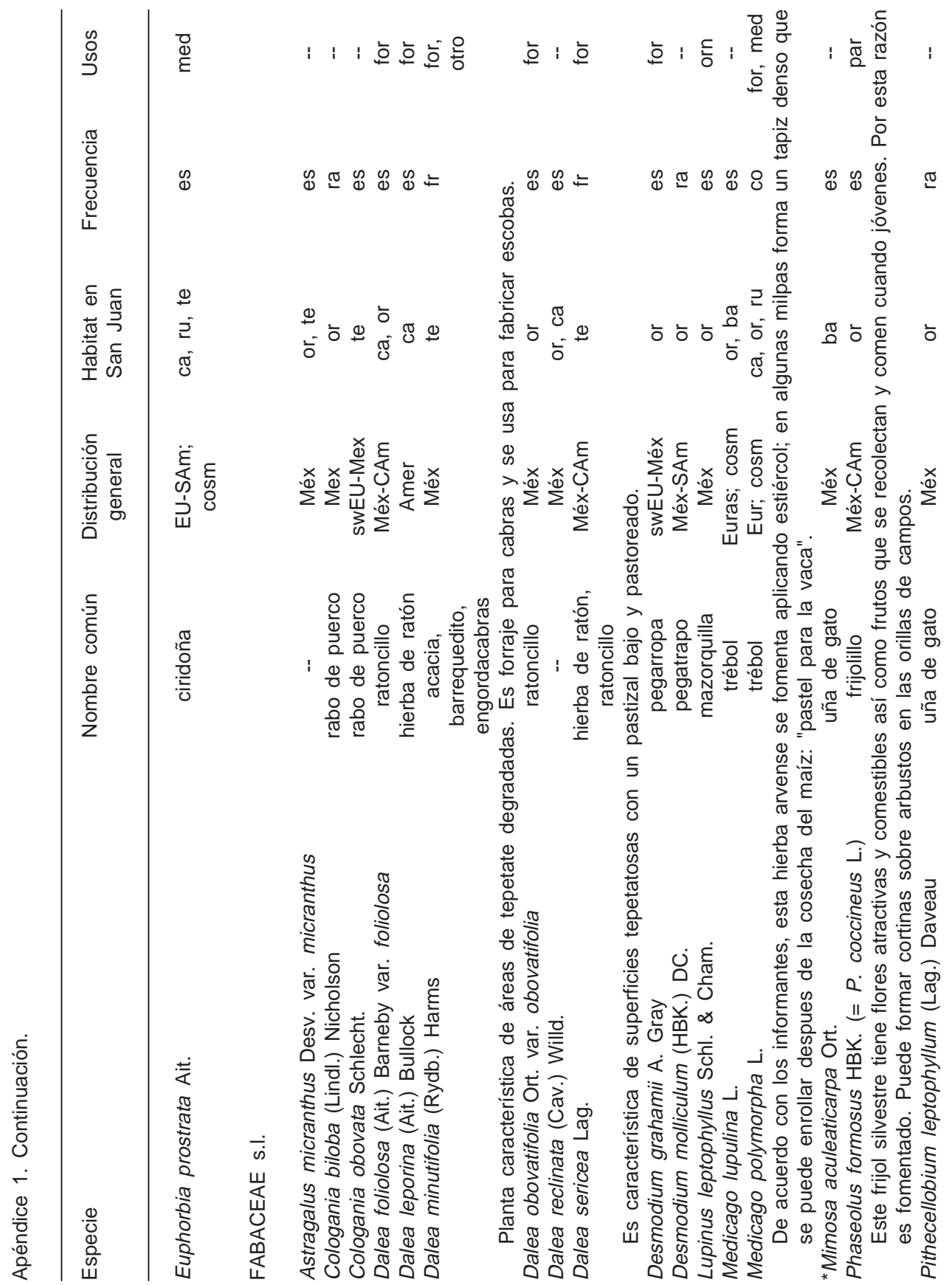




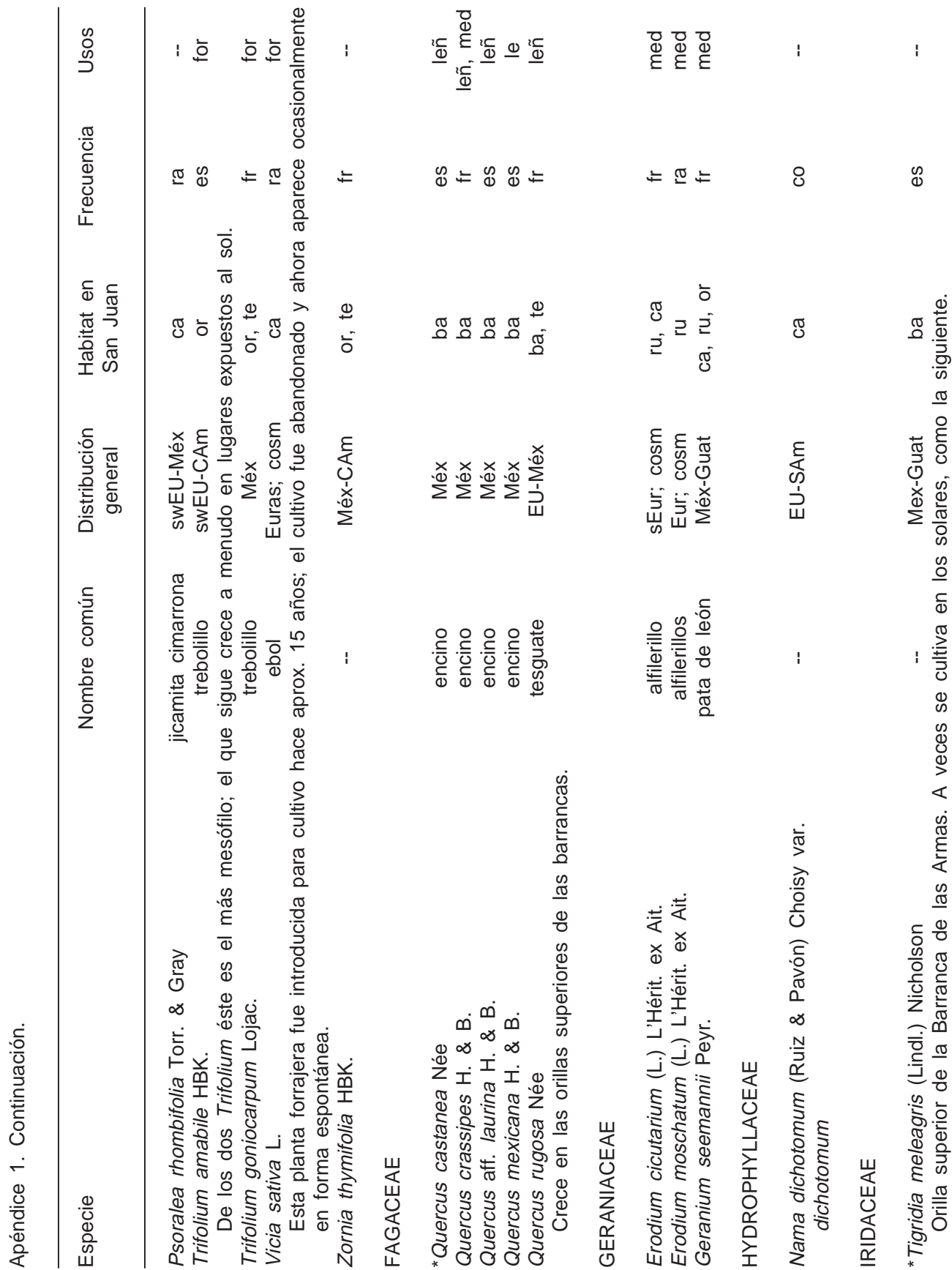


Vibrans: Plantas Vasculares Silvestres en San Juan Quetzalcoapan, Tlaxcala

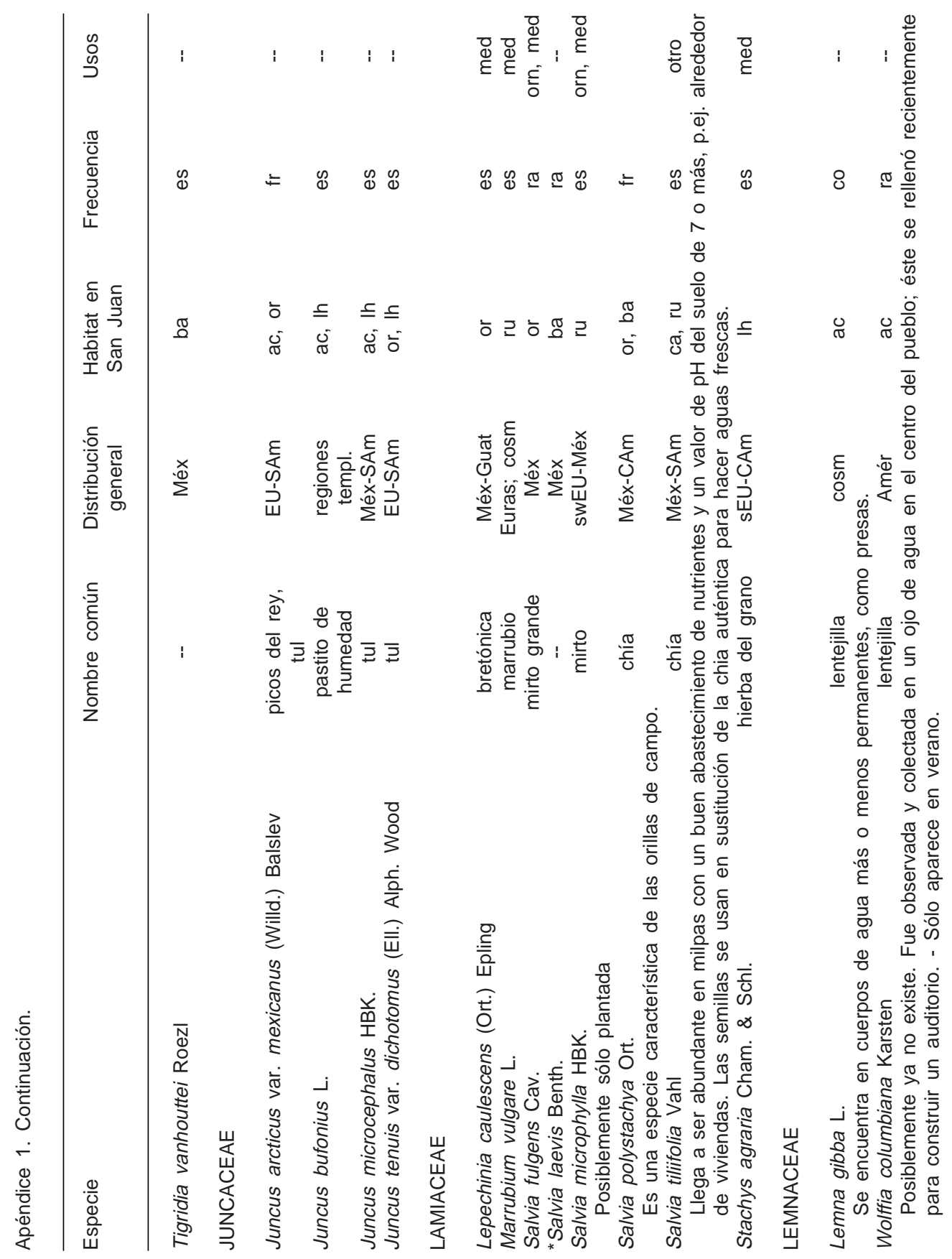




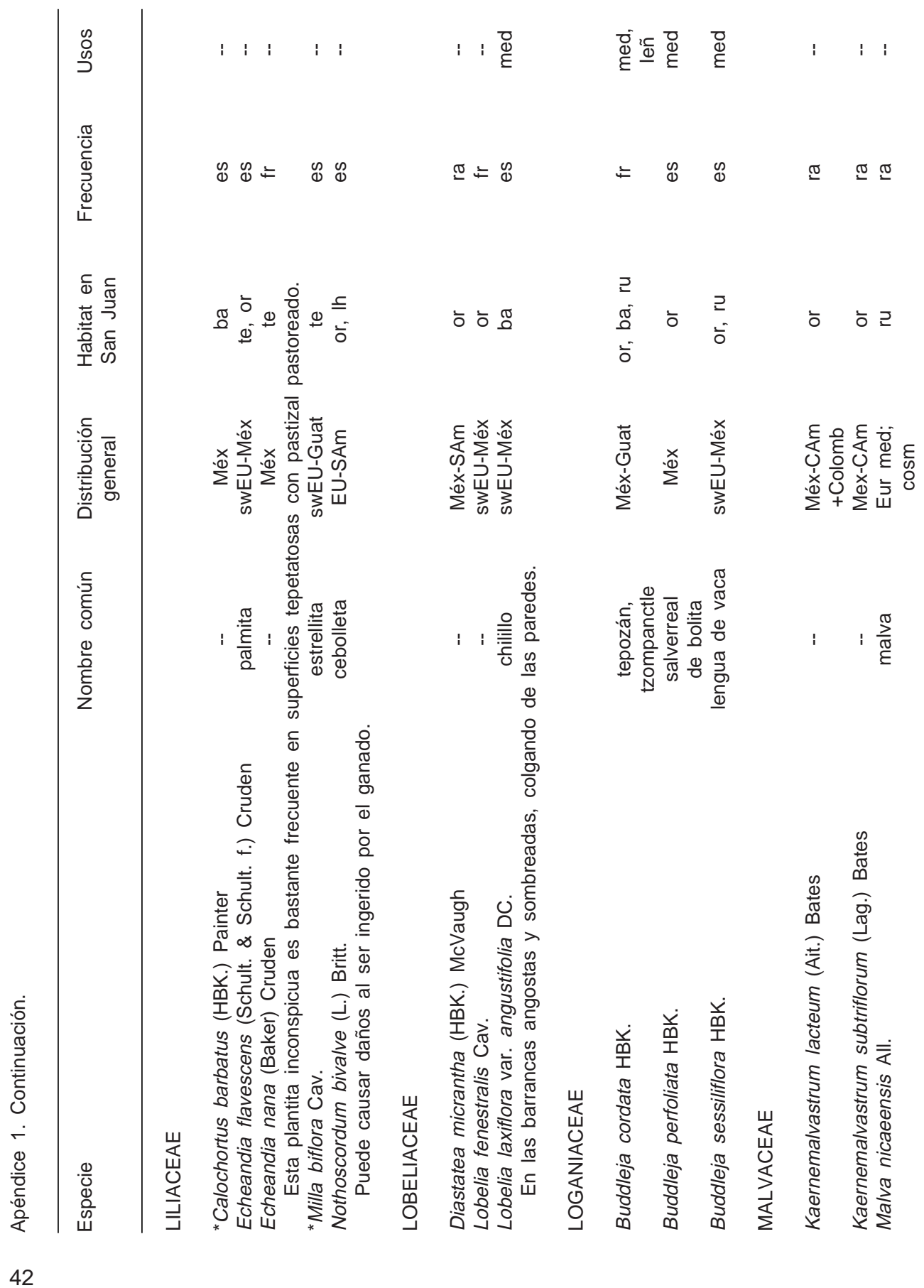


Vibrans: Plantas Vasculares Silvestres en San Juan Quetzalcoapan, Tlaxcala

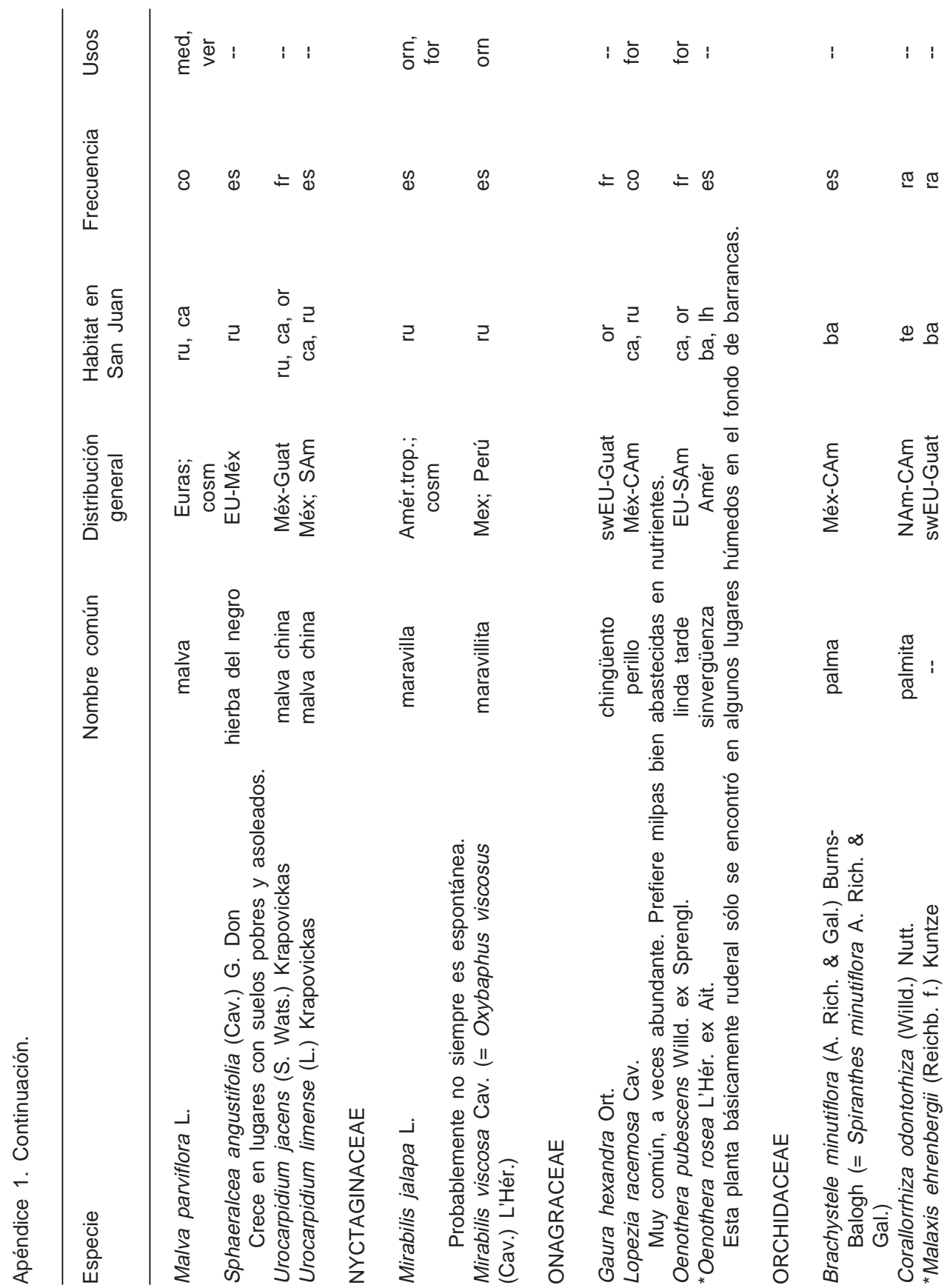




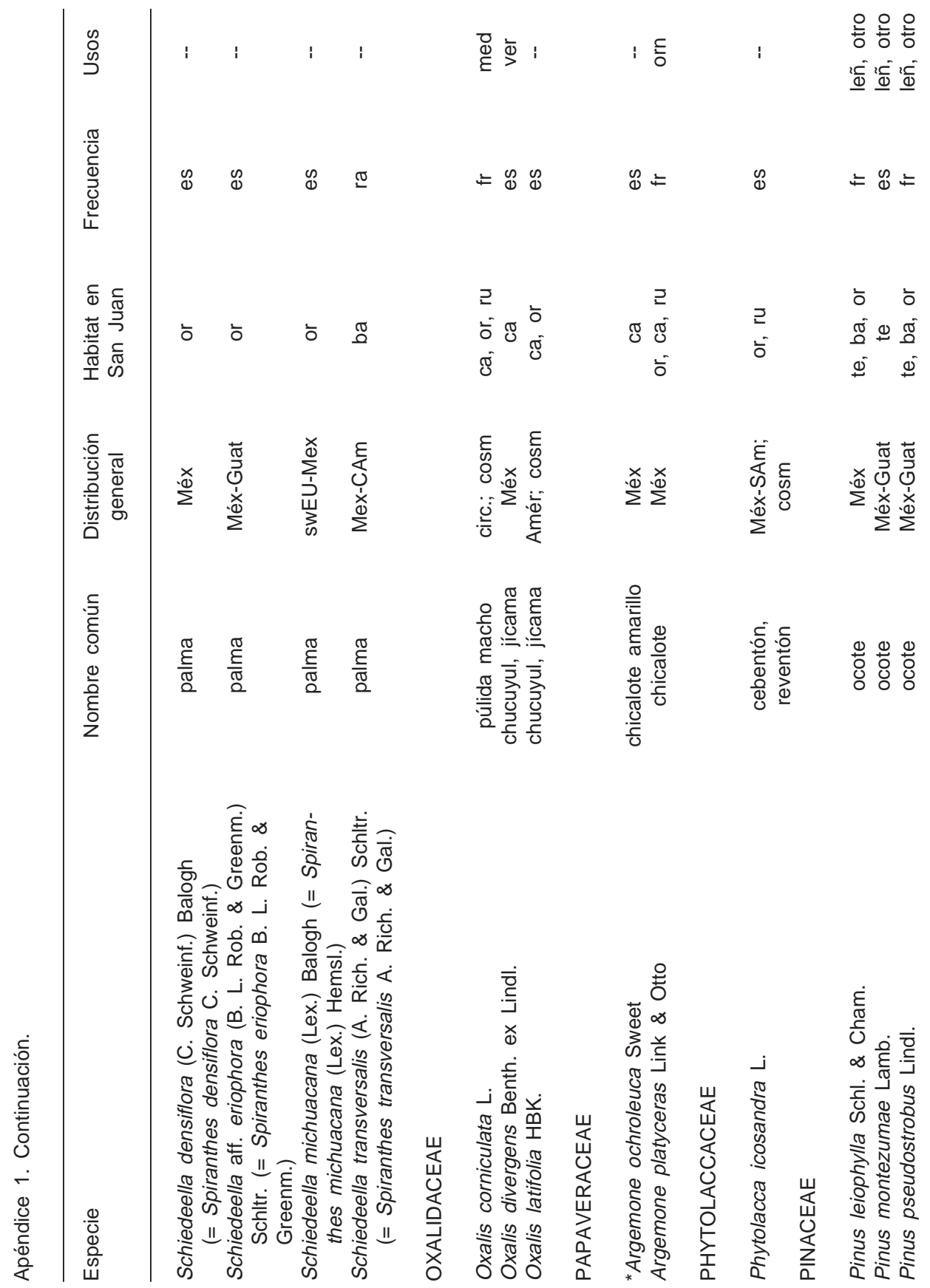


Vibrans: Plantas Vasculares Silvestres en San Juan Quetzalcoapan, Tlaxcala

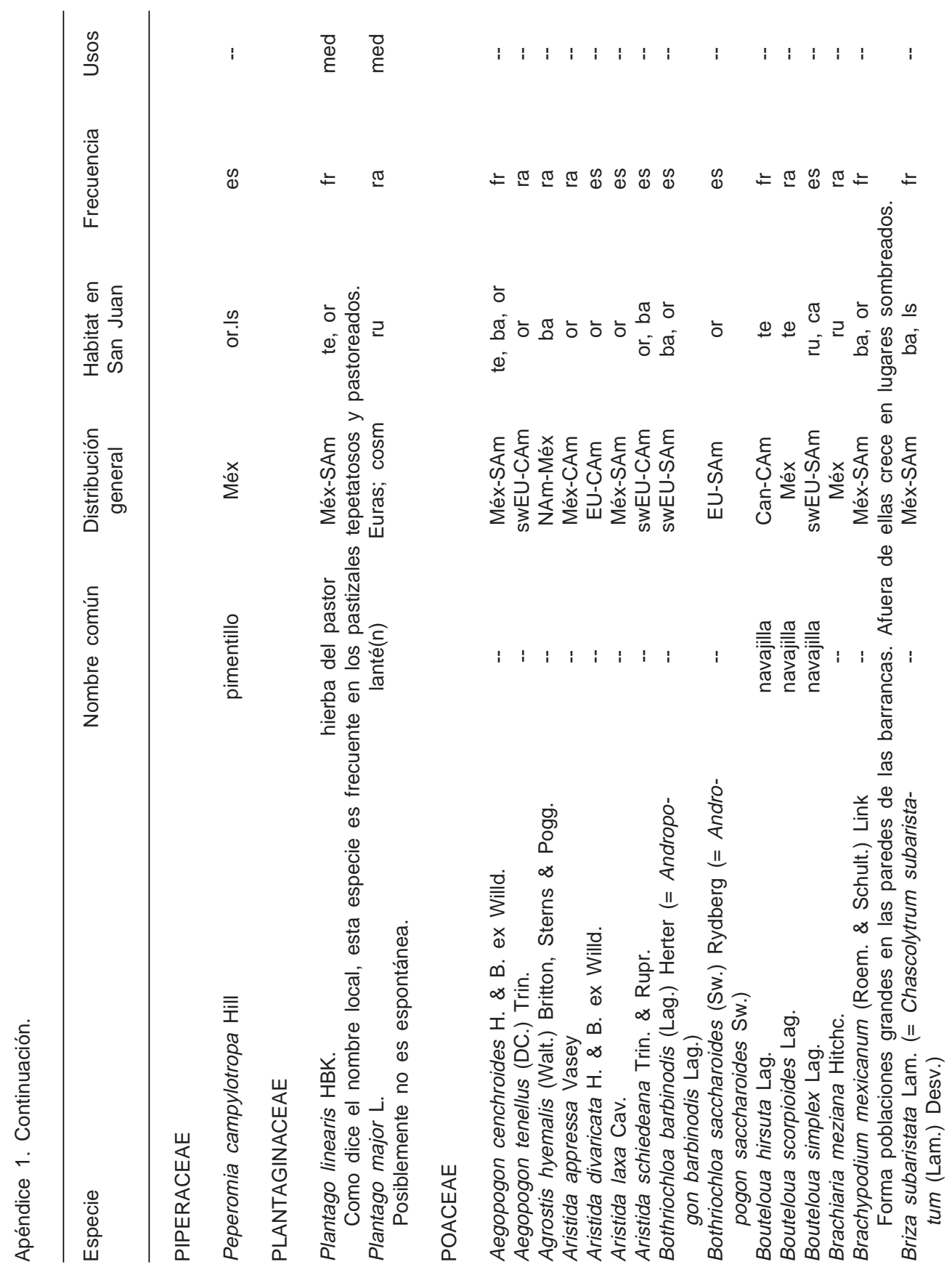




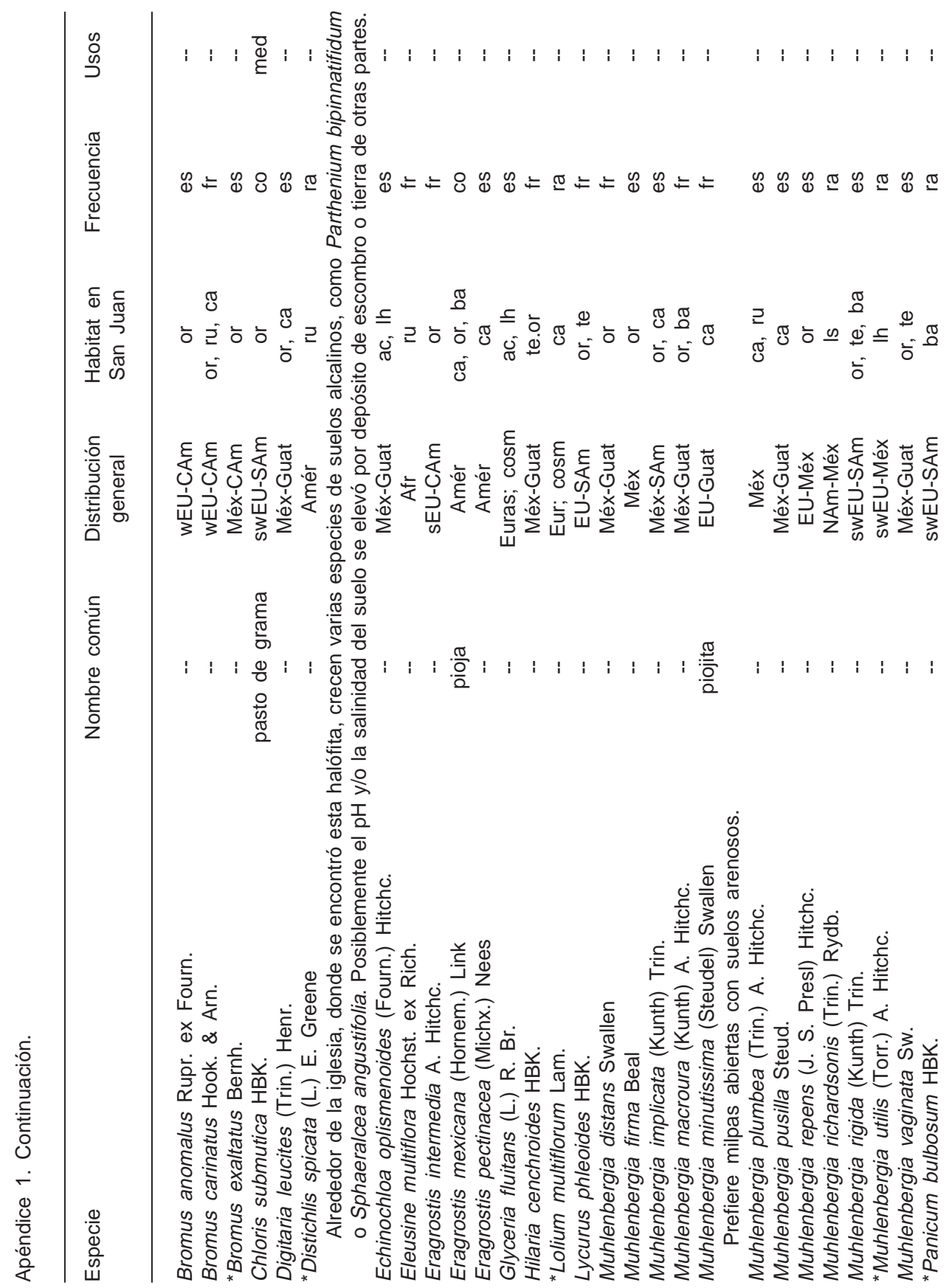


Vibrans: Plantas Vasculares Silvestres en San Juan Quetzalcoapan, Tlaxcala

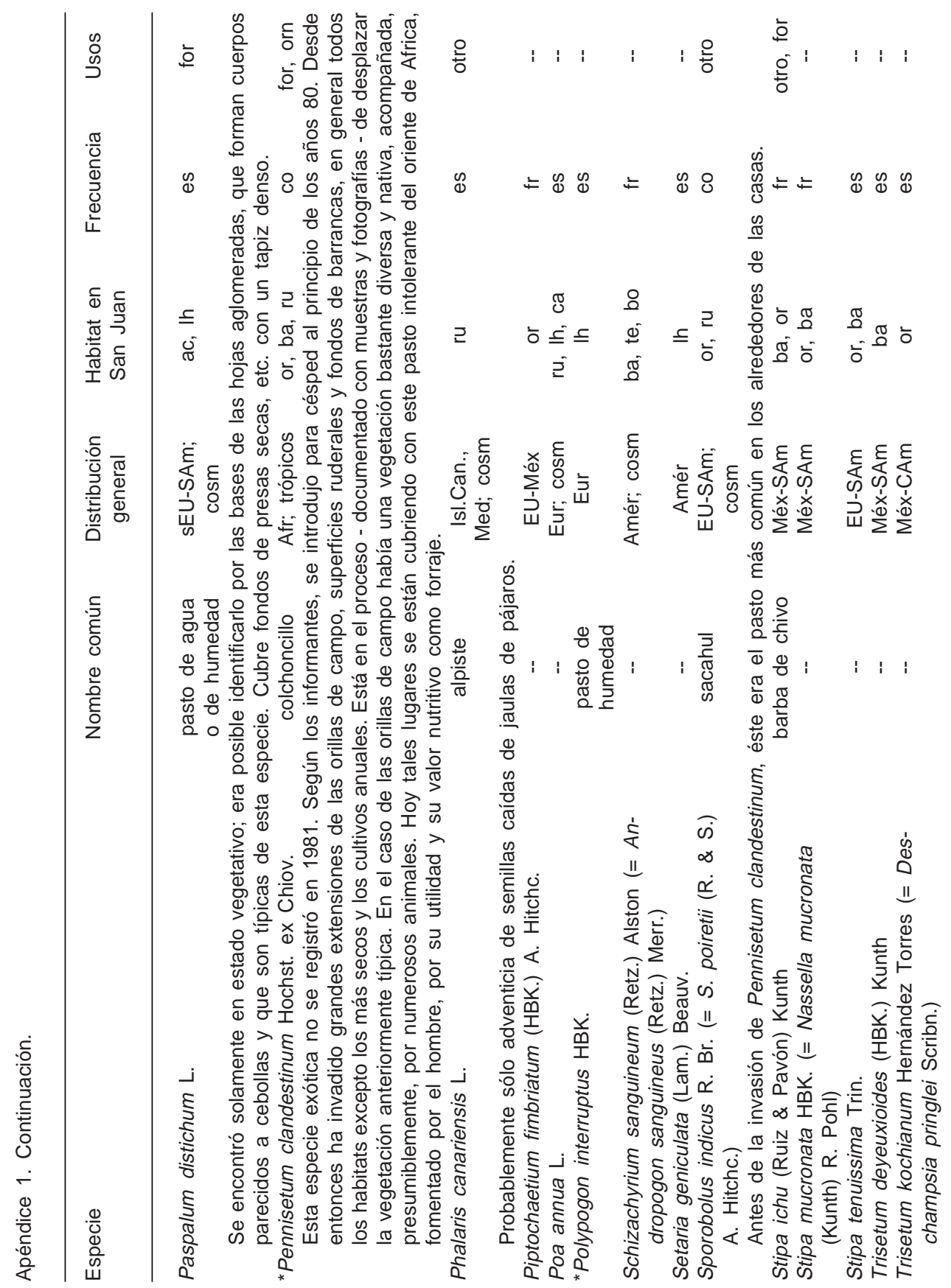




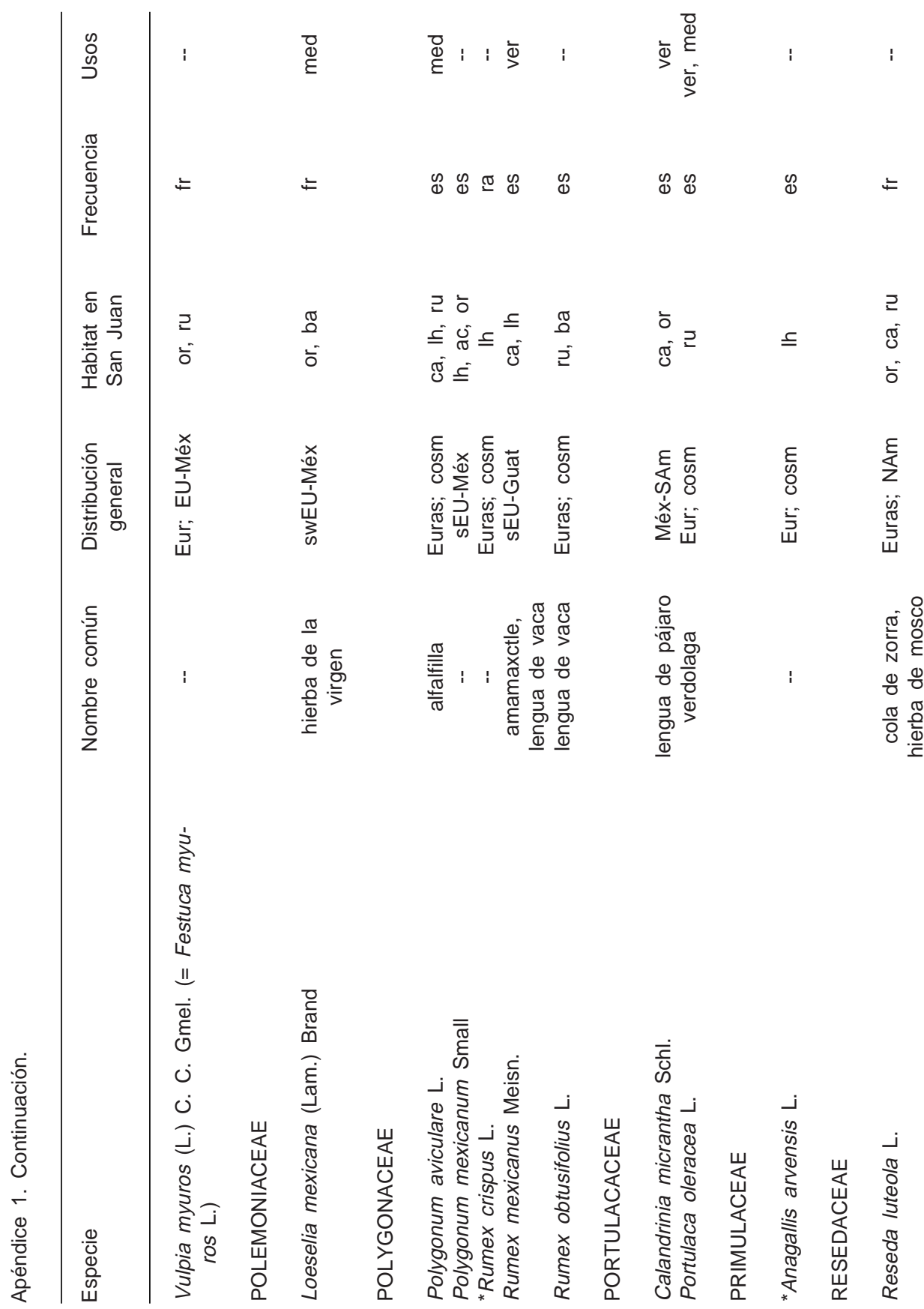


Vibrans: Plantas Vasculares Silvestres en San Juan Quetzalcoapan, Tlaxcala

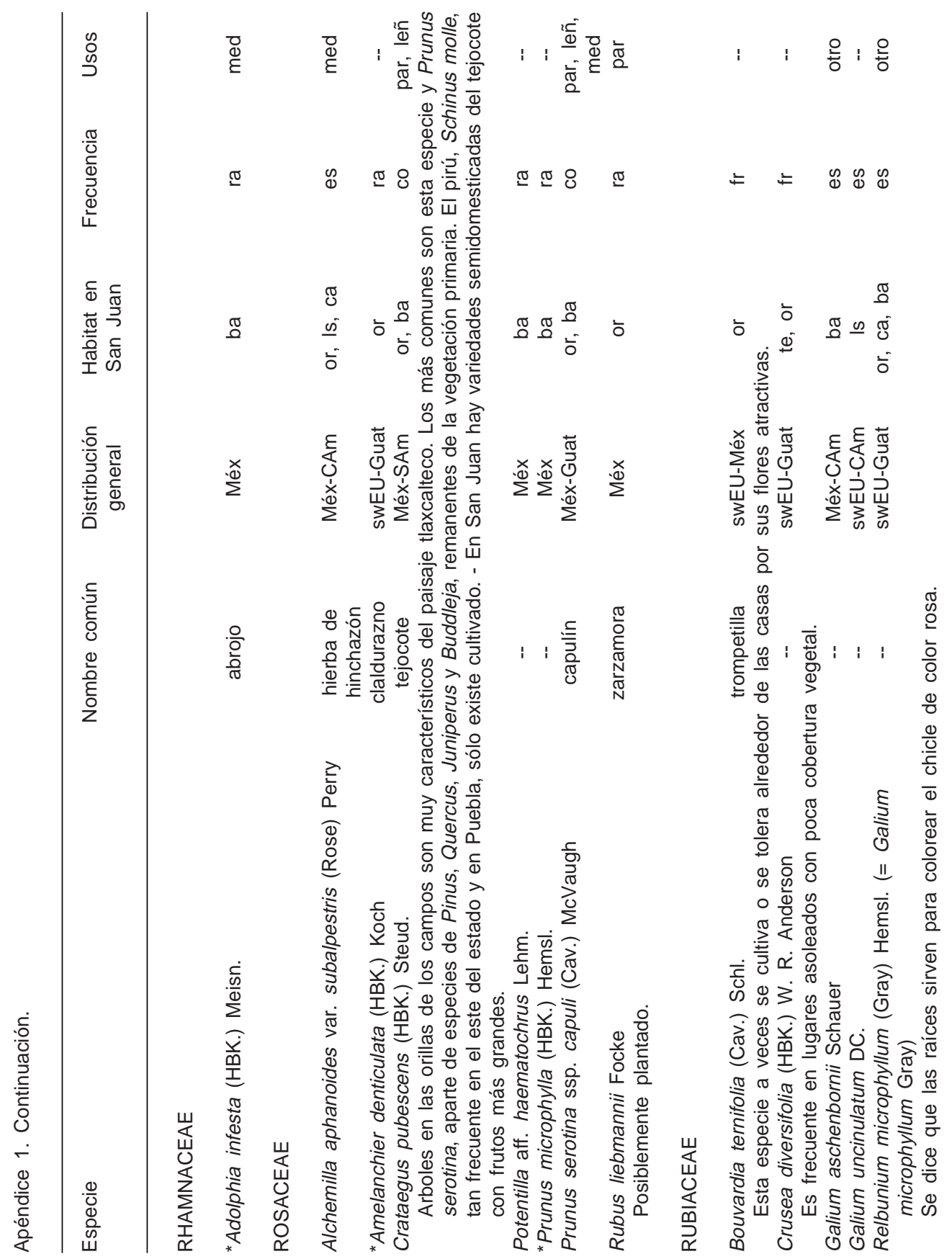




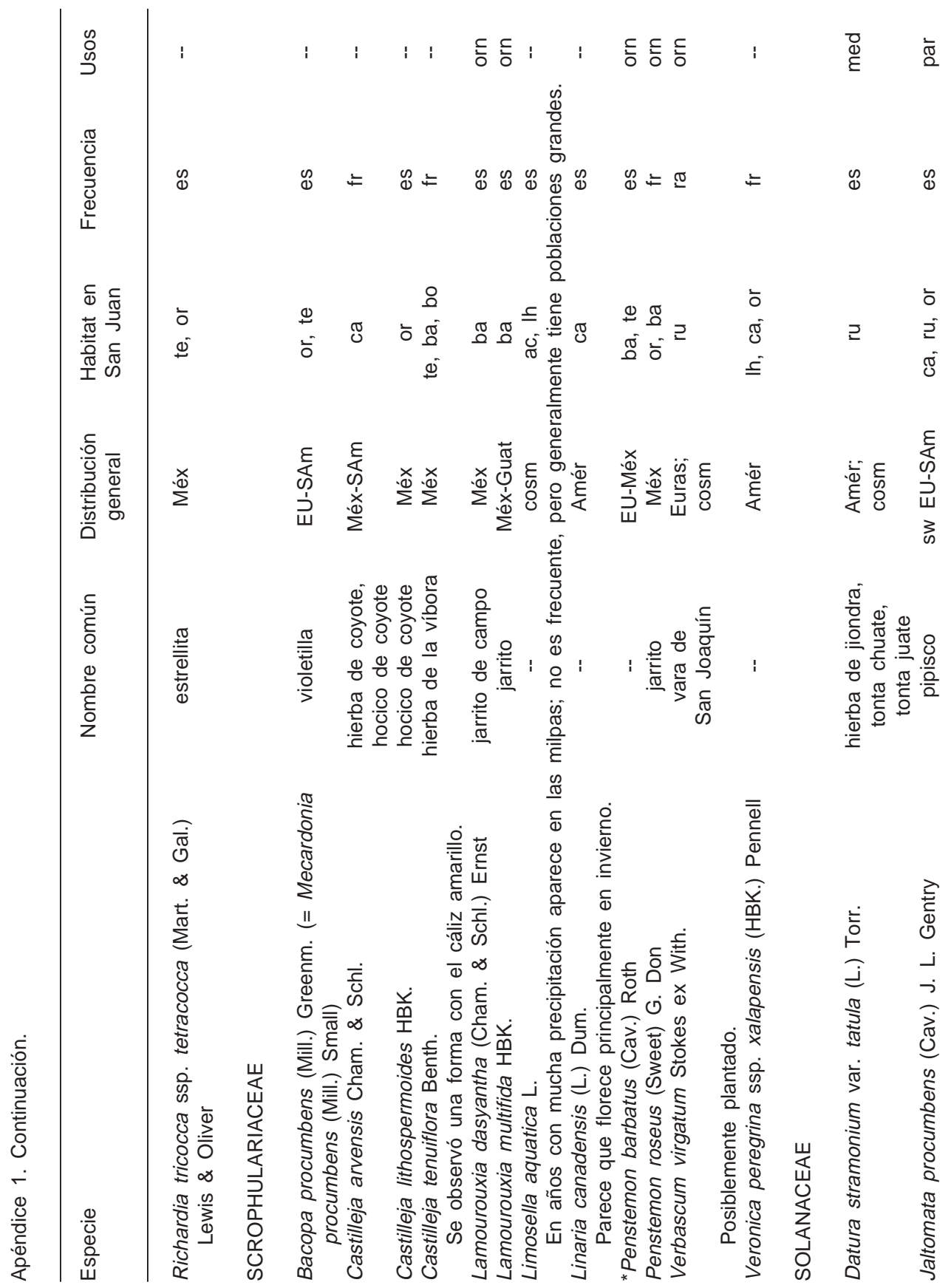


Vibrans: Plantas Vasculares Silvestres en San Juan Quetzalcoapan, Tlaxcala

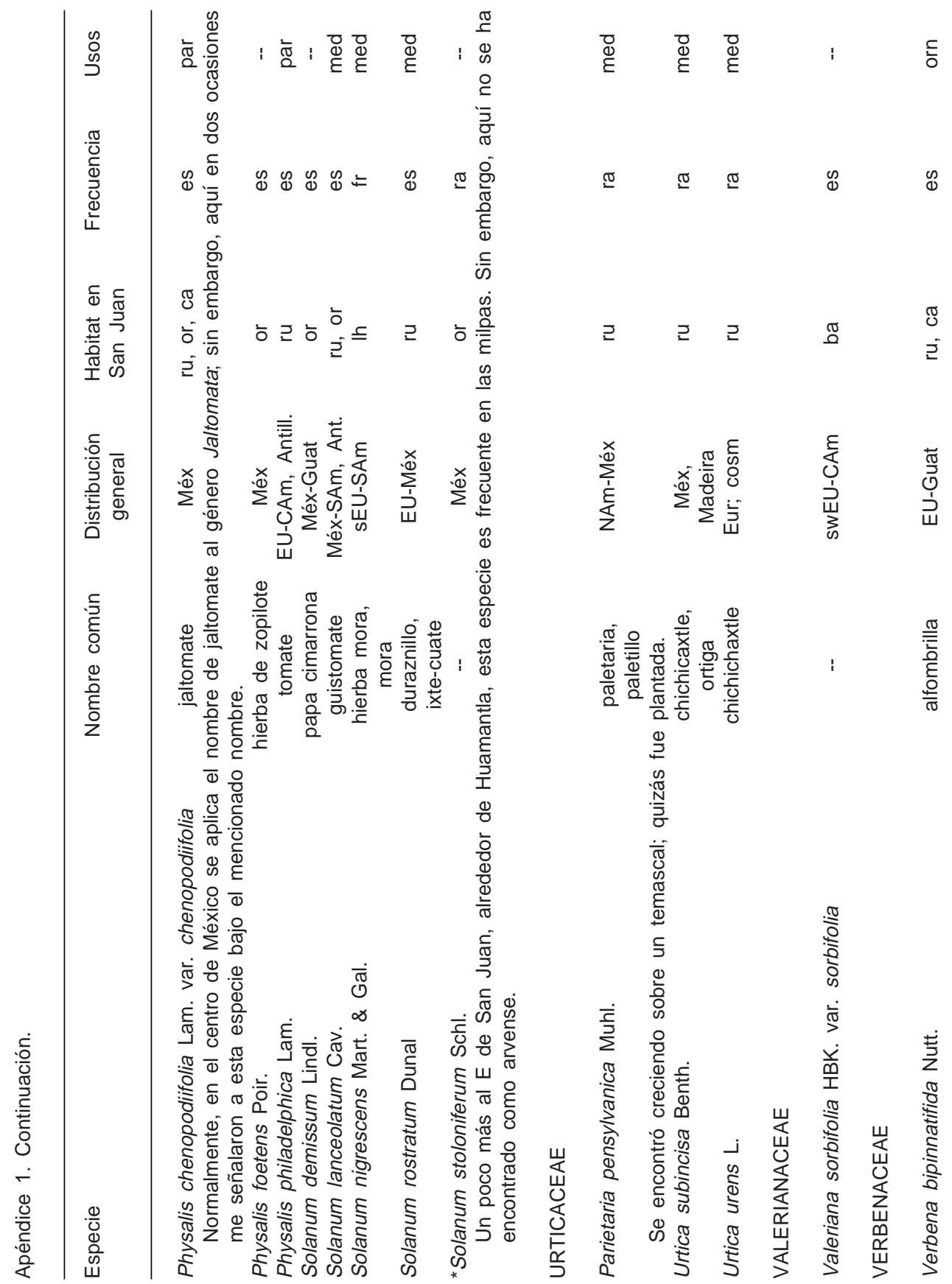



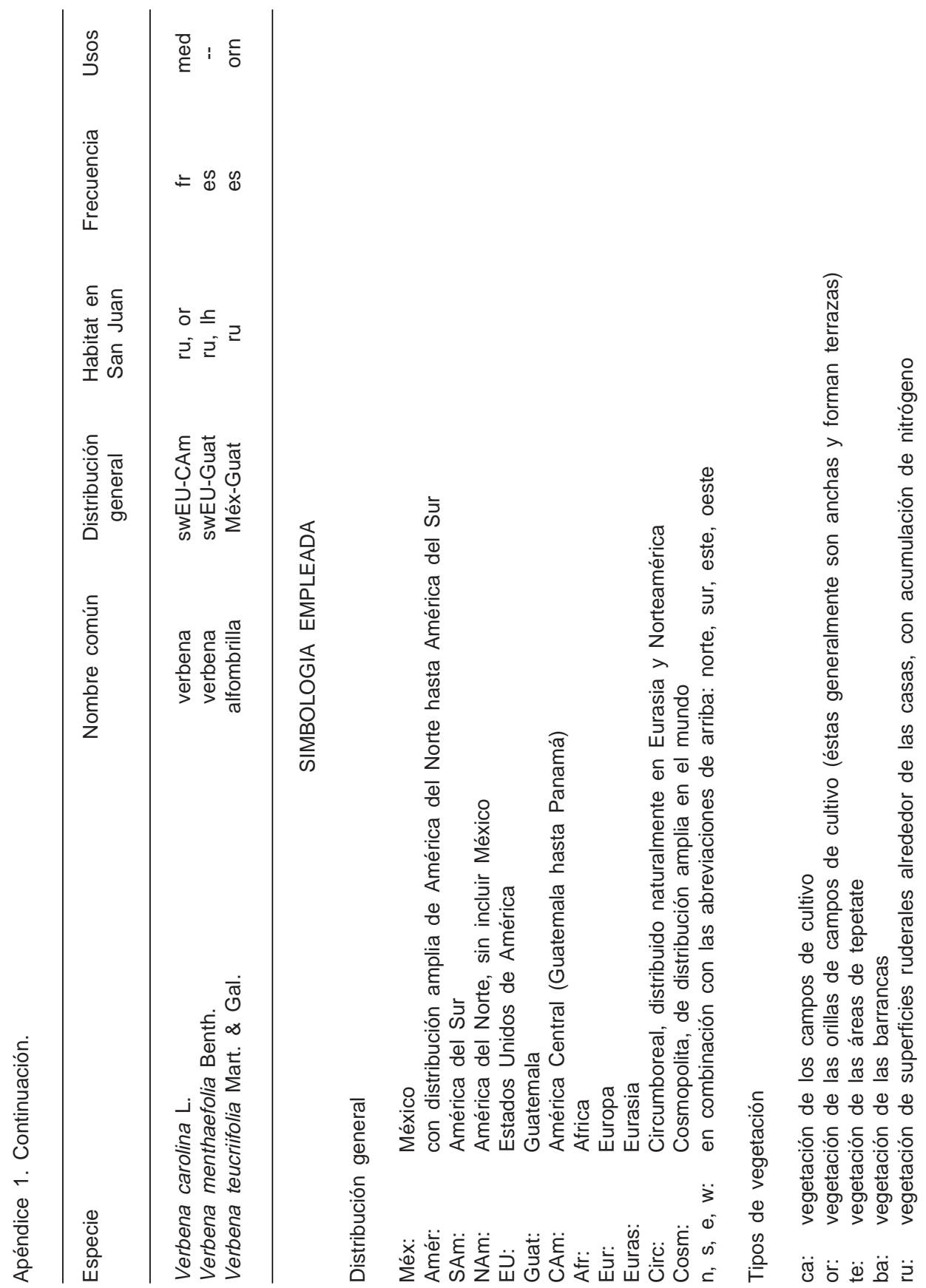
Vibrans: Plantas Vasculares Silvestres en San Juan Quetzalcoapan, Tlaxcala

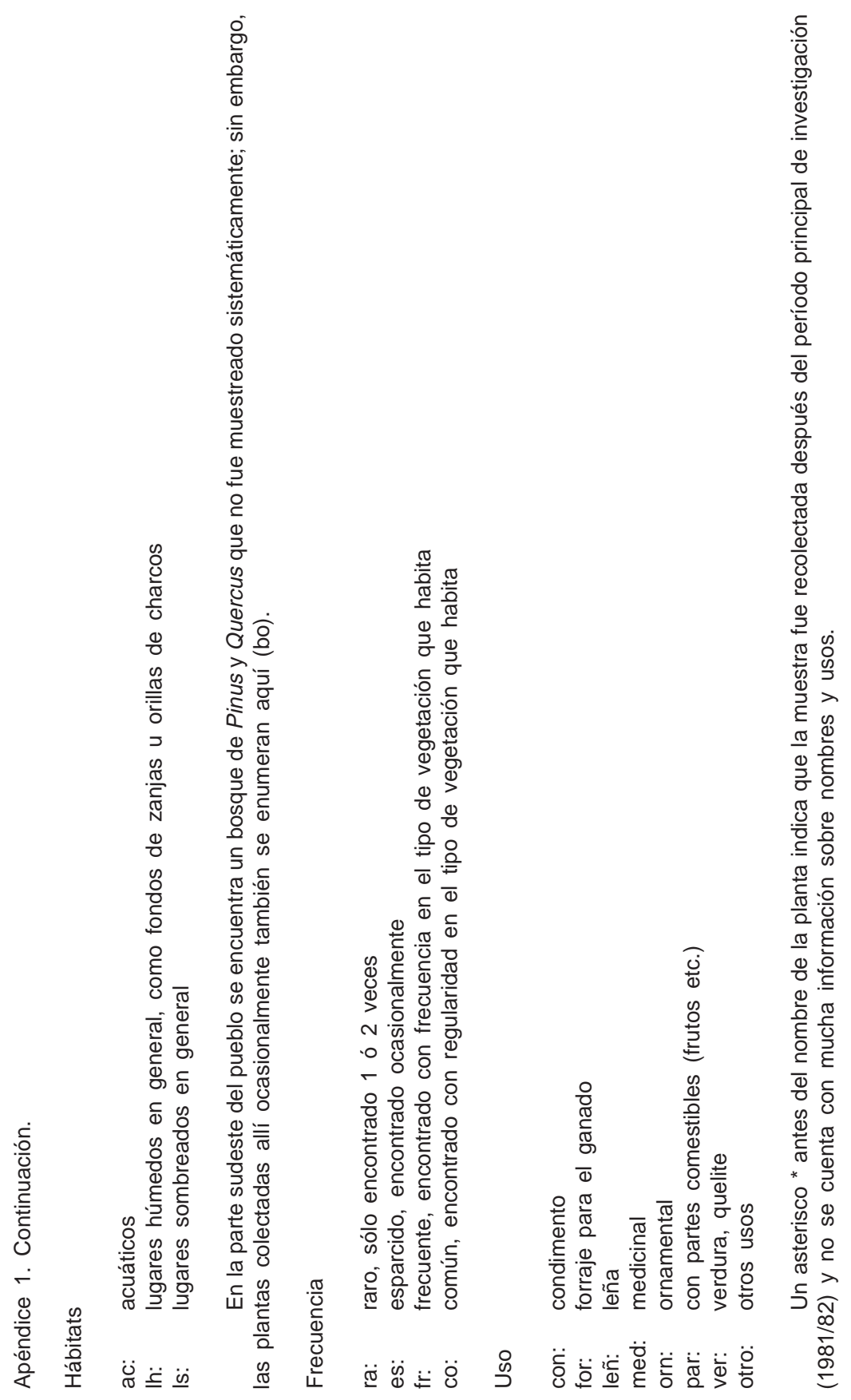


Apéndice 2. Registro de colectas indicando las temporadas de floración y los herbarios donde se encuentra depositado un duplicado.

\section{PTERIDOPHYTA}

ASPLENIACEAE: Asplenium monanthes: 897:28-1-82; 912: 28-1-82 (MEXU); 1081:26-9-82 DRYOPTERIDACEAE: Cystopteris fragilis: 523:21-10-81 (MEXU); Phanerophlebia nobilis: 1002:14-3-82

POLYPODIACEAE: Pleopeltis polylepis: 865:17-12-81; 1216:10-10-82 (MEXU); Polypodium plebeium: 400b:14-10-81; 991:13-3-82 (B, MEXU)

PTERIDACEAE: Adiantum poiretii: 224a:20-6-81 (ENCB, MEXU); 901:28-1-82 (B); Cheilanthes hirsuta: 400d:14-10-81 (MEXU); 688:4-11-81; 728:10-11-81; Cheilanthes microphylla: 1074:26-9-82 (MEXU); Cheilanthes myriophylla: 614:29-10-81; 623:3010-81; 687:4-11-81; 910:28-1-82; 1213:10-10-82 (MEXU); Mildella intramarginalis: 2579:29-1-89; Notholaena aurea: 446:18-10-81 (B); 547:22-10-81 (ENCB, MEXU); 613:29-10-81; 682:4-11-81; 896:28-1-82; Notholaena sinuata: 612:29-10-81 (MEXU); 1206:10-10-82 (B); Pellaea cordifolia: 400c:14-10-81; 1210:10-10-82 (B, MEXU); Pellaea ternifolia: 400a:14-10-81 (MEXU); 686:4-11-81

SELAGINELLACEAE: Selaginella rupestris: 385:14-10-81 (MEXU)

\section{SPERMATOPHYTA}

AMARANTHACEAE: Alternanthera caracasana: 645:30-10-81; 3767:31-5-92; Amaranthus hybridus: 162:177-6-81 (ENCB, MEXU); 247:18-9-81; 270:26-9-81; 330:7-10-81; 332:7-10-81; 503b:18-10-81; 637:30-10-81; 739:10-11-81; 773:11-11-81; 776:11-1181; 777:11-11-81 (B); 1141:4-10-82; Gomphrena pringlei: 847:18-11-81 (MEXU); 1079:26-9-82 (B); Guilleminea densa: 427:15-10-81; 586:29-10-81; 646:30-10-81 (MEXU); 3768:31-5-92

AMARYLLIDACEAE: Hymenocallis acutifolia: 3777:31-5-92; Zephyranthes brevipes: 3640:44-92; Zephyranthes sessilis: 3734:30-5-92

ANACARDIACEAE: Rhus standleyi: 993:13-3-82

APIACEAE: Apium leptophyllum: 605:29-10-81; 1194:7-10-82 (ENCB, MEXU); Arracacia tolucensis: 3758:30-5-92; Daucus montanus: 201:19-6-81; 594:29-10-81; 618:29-1081; 700:4-11-81 (B); 818:12-11-81 (ENCB, MEXU); Eryngium carlinae: 189:19-6-81 (MEXU); 527:21-10-81; 534:21-10-81 (veget.); 960:29-1-82 (veget.); Prionosciadium thapsoides: $522: 21-10-81$ (ENCB, MEXU)

ASCLEPIADACEAE: Asclepias linaria: 139:16-6-81; 1030:22-9-82 (B, ENCB, MEXU); Asclepias notha: 185:19-6-81 (MEXU); 3754:30-5-92; Matelea sp.: 202:20-6-81; Metastelma angustifolium: 218:20-6-81 (MEXU); 444:18-10-81; 1122:26-9-82 (B, MEXU); 1130:27-9-82 (B); 3637:4-4-92

ASTERACEAE: Achillea millefolium: 186:19-6-81; 696:4-11-81 (veget.); 733:10-11-81 (veget.); 828:12-11-81 (veget.); 857:19-11-81 (ENCB, MEXU); 861:19-11-81; Achyropappus anthemoides: 659:2-11-81 (ENCB, MEXU); Acourtia alamanii: 1143:410-82 (ENCB, MEXU); Aphanostephus ramosissimus: 850:18-11-81; 871:27-1-82; 963:29-1-82 (MEXU); 967:29-1-82 (B); 3740:30-5-92; Archibaccharis serratifolia: 
1144:4-10-82 (ENCB, MEXU); Artemisia ludoviciana: 986:23-2-82 (veget.); 1164:510-82 (B, ENCB, MEXU); 1177:6-10-82; Aster moranensis: 860:19-11-81 (B, ENCB, MEXU); Aster subulatus: 194:19-6-81 (B); 1157:5-10-82 (ENCB, MEXU); Baccharis conferta: 890:28-1-82 (ENCB, MEXU); Baccharis pteronioides: 620:30-10-81 (veget.); 1006:14-3-82 (B, MEXU); 1123:26-9-82; Baccharis salicifolia: 793:11-11-81 (ENCB, MEXU); Bahia xylopoda: 136:16-6-81 (ENCB, MEXU); 1209:10-10-82; 3747:30-5-92; Bidens anthemoides: 798:11-11-81; 849:18-11-81; 970:29-1-82; 1013:15-3-82 (B, MEXU); Bidens aurea: 747:10-11-81; 794:11-11-81 (ENCB, MEXU); 1182:6-10-82 (ENCB, MEXU); 1183:6-10-82 (ENCB, MEXU; ejemplar con flores liguladas blancas); 1197:7-10-82; Bidens odorata: 155:17-6-81; 159:17-6-81; 246:18-9-81; 268:26-9-81;

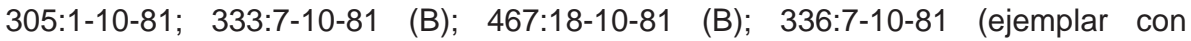
deformaciones); 600:29-10-81; 616:29-10-81 (B); 621b:30-10-81; 676:3-11-81; 677:3-11-81; 835:12-11-81; 839:12-11-81; 1031:22-9-82 ((ENCB, MEXU, ejemplar con deformaciones); Brickellia secundiflora: 393:14-10-81 (B, ENCB, MEXU); 820:1211-81 (MEXU); Brickellia veronicifolia: 397:14-10-81 (ENCB, MEXU); 1220:10-10-82; Cirsium aff. acantholepis: 3840:16-8-92; Conyza canadensis: 296:1-10-81 (MEXU); 435:18-10-81; 601:29-10-81; 602:29-10-81; 624:30-10-81; 713:9-11-81; 714:9-11-81; 838:12-11-81; 975:29-1-82 (B); Conyza coronopifolia: 223:20-6-81; 368:8-10-81; 617:29-10-81; 644:30-10-81; 715:9-11-81; 837:12-11-81; 947:29-1-82; 948:29-1-82; 957:29-1-82; 972:29-1-82 (B, MEXU); 1104:27-9-82; 3658:16-4-92; Conyza filaginoides: 546:22-10-81 (B); 1071:25-9-82; 1080:26-9-82; 1113:26-9-82 (MEXU); 3866:16-8-92; Conyza sophiifolia: 187:19-6-81 (B); 939:29-1-82 (MEXU); 961:29-1-82; 2595:29-189; 3656:16-4-92; Cosmos bipinnatus: 150:17-6-81 (B, MEXU); 506:18-10-81; Dahlia coccinea: 1075:26-9-82 (MEXU, sólo frutos); 3653:16-4-92; 3830:15-8-92; Dugesia mexicana: 2680:9-7-89; Dyssodia papposa: 487:18-10-81; 627:30-10-81 (B); 719:911-81 (MEXU); Dyssodia pinnata: 209:20-6-81 (ENCB, MEXU); Erigeron karvinskianus: 866:17-12-81 (B, MEXU); 880:28-1-82; Erigeron longipes: 156:17-6-81; 178:19-6-81 (ENCB, MEXU); 311:1-10-81 (veget.); 423:15-10-81 (veget.); 453:18-10-81 (veget.); 625:30-10-81 (veget.); 915:28-1-82; 942:29-1-82; 998:14-3-82 (B); Eupatorium deltoideum: 3844:15-8-92; Eupatorium glabratum: 859:19-11-81; 873:27-1-82 (ENCB, MEXU); 1175:6-10-82; 2597:29-1-89; Eupatorium irrasum: 138:16-6-81; 3746:30-592; Eupatorium petiolare: 878:28-1-82 (ENCB, MEXU); Eupatorium pulchellum: 619:30-10-81; 813:12-11-81 (B); 1170:5-10-82 (ENCB, MEXU); Eupatorium schaffneri: 510:19-10-81 (ENCB, MEXU); Eupatorium scorodonioides: 141:16-6-81; Florestina pedata: 158:17-6-81 (ENCB, MEXU); 274:26-9-81 (B); 962:29-1-82; Galinsoga parviflora: 248:18-9-81; 475:18-10-81 (MEXU); 632:30-10-81; 735:10-11-81 (B); Galinsoga quadriradiata: 1083:26-9-82 (B, MEXU); Gnaphalium americanum: 1139:27-9-82 (MEXU); 3669:17-4-92; Gnaphalium bourgovii: 348:7-10-81; 1178:610-82 (ENCB, MEXU); 1192:6-10-82; Gnaphalium conoideum: 127:15-6-81; 691:411-81; 699:4-11-81; 1119:26-9-82; 1186:6-10-82; 1219:10-10-82; Gnaphalium oxyphyllum: 281:30-9-81; Gnaphalium purpurascens: 429:16-10-81 (MEXU); 3861:168-92; Gnaphalium semiamplexicaule: 465:18-10-81; 1212:10-10-82 (MEXU); 3775:315-92; Gnaphalium sphacilathum: 3741:31-5-92; Gnaphalium stagnale: 729:10-11-81; 846:18-11-81 (MEXU); 964:29-1-82; Gnaphalium stramineum: 941:29-1-82 (MEXU); Haplopappus venetus: 370:14-10-81 (ENCB, MEXU); 936:29-1-82; 937:29-1-82 (B); 
Heterosperma pinnatum: 514:21-10-81 (B, ENCB, MEXU); 550:22-10-81; Heterotheca inuloides: 122: 15-6-81; 152:17-6-81 (ENCB, MEXU); 531:21-10-81; 533:21-10-81; 626:30-10-81; 930:29-1-82 (B); 3651:16-4-92; Hieracium schultzii: 2660:8-7-89; 2690:9-7-89; 3832:15-8-92; Hybridella globosa: 2672:9-7-89; Kuhnia rosmarinifolia: 872:27-1-82 (B, ENCB, MEXU); 994:13-3-82; 1016:22-9-82; 3635:4-4-92; Melampodium bibracteatum: 358:8-10-81; 754:10-11-81; 1198:7-10-82 (MEXU); Montanoa tomentosa: 1127:27-9-82 (MEXU); Parthenium bipinnatifidum: 472:18-10-81 (ENCB, MEXU); 585:29-10-81 (B); 636:30-10-81; Pinaropappus roseus: 123:15-6-81; 275:26-9-81 (veget.); 319:2-10-81; 326:2-10-81 (veget.); 503:18-10-81; 932:29-1-82; 938:29-1-82 (ENCB, MEXU); Piqueria trinervia: 200:19-6-81; 620b:30-10-81 (B, ENCB, MEXU); Sabazia humilis: 411:15-10-81 (B, ENCB, MEXU); 840:12-11-81; 1124:26-9-82; Sanvitalia procumbens: 484:18-10-81 (B); 634:30-10-81 (MEXU); Schkuhria pinnata: 195:19-6-81 (MEXU); 362:8-10-81; 750:10-11-81 (B); Senecio peltiferus: 1110:27-982 ((MEXU); Senecio salignus: 372:14-10-81 (sin flor); 876:28-1-82 (con botones); 988:23-2-82 (B, ENCB, MEXU); Simsia amplexicaulis: 157:17-6-81 (MEXU); 245:189-81 (MEXU); 381:14-10-81 (MEXU); 410:15-10-81 (MEXU); 413:15-10-81 (MEXU); 516:21-10-81 (MEXU); 608:29-10-81 (MEXU); 674:3-11-81 (MEXU); 781:11-11-81 (MEXU); 840b:12-11-81 (MEXU); 931:29-1-82; 1033:22-9-82 (forma deformada); Sonchus asper: 782a:11-11-81; 966:29-1-82 (B); 968:29-1-82 (MEXU); 3681:17-492; Sonchus oleraceus: 254:24-9-81 (B); 458:18-10-81 (ENCB, MEXU); 633:30-1081; 745:10-11-81; 782:11-11-81; 823b:12-11-81; 895:28-1-82; Spilanthes oppositifolia: 1181:6-10-82; Stevia nepetifolia: 371:14-10-81 (ENCB, MEXU); Stevia ovata: 821:1211-81; 832:12-11-81; 3867:16-8-92; Stevia pilosa: 1044:24-9-82 (MEXU); Stevia salicifolia: 1153:4-10-82 (ENCB, MEXU); Stevia subpubescens: 392:14-10-81; 855:18-11-81; 990:13-3-82 (B, ENCB, MEXU); 1176:6-10-82; 2596:29-1-89; Stevia viscida: 215:20-6-81; Tagetes coronopifolia: 443:18-10-81 (MEXU); 611:29-10-81 (MEXU); 1160:5-10-82; Tagetes erecta: probablemente escapada de cultivo. 597:2910-81 (MEXU); 709:9-11-81; Tagetes lunulata: 389:14-10-81 (MEXU); 395:14-10-81 (MEXU); 464:18-10-81 (MEXU); 502:18-10-81 (MEXU); 629:30-10-81 (MEXU); 708:911-81 (MEXU); 710:9-11-81 (MEXU); 1149:4-10-82 (ENCB, MEXU); Tagetes micrantha: 295:1-10-81 (MEXU); 1043:24-9-82 (B); 3875:16-8-92; Taraxacum officinale: 470:18-10-81; 628:30-10-81 (MEXU); 736:10-11-81; Tridax coronopifolia: 145:16-6-81 (B); 146:16-6-81; 272:26-9-81; 384:14-10-81 (MEXU); Verbesina virgata: 391:14-10-81; 580:29-10-81 (B); 841:18-11-81 (ENCB, MEXU)

BEGONIACEAE: Begonia gracilis: 3843:16-8-92

BRASSICACEAE: Brassica rapa: 160:17-6-81; 238:18-9-81; 285:1-10-81; 954:29-1-82 (B, MEXU); Capsella bursa-pastoris: 494:18-10-81; Coronopus didymus: 2594:29-1-89; Descurainia impatiens: 457:18-10-81 (B, ENCB, MEXU); 584:29-10-81 (veget.); 797:11-11-81; 913:28-1-82; 946:29-1-82 (veget.); 959:29-1-82 (veget.); 980:22-2-82; Descurainia virletii: 3362:10-11-90; Halimolobos berlandieri: 2685:9-7-89; Lepidium oblongum: 383:14-10-81; 590:29-10-94; Lepidium schaffneri: 491:17-10-81; Lepidium virginicum: 153:17-6-81; 635:30-10-81; 685:2-11-81; 784:11-11-81; 3675:17-4-92; 3774:31-5-92; 3755:31-5-92; Raphanus raphanistrum: 161:17-6-81 (ENCB); 241:189-81 (MEXU); 943:29-1-82; Rorippa mexicana: 196:19-6-81 (MEXU); 360:8-10-81; 3672:17-4-92;3778:31-5-92 
Vibrans: Plantas Vasculares Silvestres en San Juan Quetzalcoapan, Tlaxcala

BROMELIACEAE: Tillandsia recurvata: 211:20-6-81; 492:18-10-81; 997:14-3-82 (B, ENCB, MEXU); Tillandsia usneoides: 212:20-6-81 (MEXU)

CACTACEAE: Las Cactáceas fueron determinadas por Jerónimo Reyes, Jardín Botánico, UNAM, con pencas vivas colectadas el 17 de abril y el 31 de mayo de 1992. No fueron herborizadas.

CALLITRICHACEAE: Callitriche terrestris: 364:8-10-81 (MEXU); Callitriche deflexa: 748:1011-81

CAPRIFOLIACEAE: Symphoricarpos microphyllus: 224b:20-6-81; 349:7-10-81; 660:2-11-81 (B, ENCB, MEXU); 1029:22-9-82 (ENCB, MEXU); 3872:16-8-92

CARYOPHYLLACEAE: Arenaria lanuginosa: 148:16-6-81; 177:19-6-81 (B, ENCB, MEXU); 512:19-10-81; 1027:22-9-82; 1072:25-9-82; Arenaria lycopodioides: 214:20-6-81 (MEXU); 525:21-10-81; Cardionema ramosissima: 665:2-11-81; 951:29-1-82; 1062:259-82; 1142:4-10-82 (B, ENCB, MEXU); Drymaria glandulosa: 244a:18-9-81 (MEXU); 1015:22-9-82; 3657:16-4-92; Drymaria laxiflora: 149:16-6-81; 978:21-2-82; Drymaria leptophylla: 299:1-10-81; 317b:2-10-81; 1189:6-10-82 (ENCB, MEXU); Drymaria malachioides: 256:25-9-81 (ENCB, MEXU); 751:10-11-81; Drymaria molluginea: 280:30-9-81; 299b:1-10-81; 1064:25-9-82 (MEXU); 1188:6-10-82 (B, ENCB, MEXU); Drymaria villosa: 244b:18-9-81; Minuartia moehringioides: 432:18-10-81 (ENCB, MEXU); 1076:26-9-82; Saponaria officinalis: 207:20-6-81; 471:18-10-81 (MEXU); 3745:30-5-92; Scleranthus annuus: 363:8-10-81; 701:4-11-81; 740:10-11-81; 1136:279-82 (MEXU); Silene laciniata: 1032:22-9-82 (B, MEXU); Spergula arvensis: 351:810-81 (B); 353:8-10-81; 599:29-10-81 (veget.); 724:10-11-81 (ENCB, MEXU); Stellaria media: 1097:27-9-82 (MEXU)

CHENOPODIACEAE: Chenopodium ambrosioides: 596:29-10-81; 772:11-11-81; 789:11-1181; 1155:5-10-82 (ENCB, MEXU); 3836:15-8-92; Chenopodium berlandieri: 307:1-1081; 769:11-11-81; 778:11-11-81; 969:29-1-82 (B); 1112:27-7-82 (MEXU); Chenopodium graveolens: 176:19-6-81 (B, ENCB, MEXU); 239:18-9-81; 240:18-9-81; 290:1-10-81; 314:2-10-81; 334:7-10-81 (B); 344a:7-10-8; Chenopodium murale: 331:7-10-81 (B); 1161:5-10-82

CISTACEAE: Helianthemum glomeratum: 394:14-10-81 (MEXU); 439:18-10-81 (B); 889:281-82

COMMELINACEAE: Commelina coelestis: 2675:9-7-89; Commelina tuberosa: 431:18-10-81; 791:11-11-81; 1059:25-9-82 (MEXU); 1093:27-9-82 (B); Tinantia erecta: 243:18-9-81; 312:2-10-81 (MEXU); Tradescantia crassifolia: 1109:27-9-82 (MEXU); 2674:9-7-89; Tripogandra purpurascens: 237:18-9-81 (B); 283:30-9-81 (MEXU); 313:2-10-81; 683:4-11-81; 783:11-11-81

CONVOLVULACEAE: Dichondra argentea: 615:29-10-81 (MEXU); 642:30-10-81; Evolvulus prostratus: 3751:31-5-92; Ipomoea purpurea: 450:18-10-81 (B, ENCB, MEXU)

CRASSULACEAE: Echeveria gibbiflora: 3661:16-4-92; Echeveria mucronata: 1185:6-10-82; Echeveria subrigida: 426:15-10-81; Sedum bourgaei: 387:14-10-81; Sedum praealtum: 473:18-10-81; 723:10-11-81; Sedum quevae: 447:18-10-81; 1054:25-9-82; Villadia batesii: 1021:22-9-82; Villadia ramossisima: 2725:27-8-89

CUCURBITACEAE: Microsechium helleri: 174:19-6-81; 504:18-10-81; 1204:7-10-82 (MEXU); Sicyos deppei: 459:18-10-81; 609:29-10-81; 1063:25-9-82 (MEXU) 
CUPRESSACEAE: Cupressus lindleyi: 565: 23-10-81; 984:22-2-82 (ENCB, MEXU); Juniperus deppeana: 369:14-10-81 (B); 434:18-10-81 (plántula); 886:28-1-82 (ENCB, MEXU); 3859:16-8-82

CYPERACEAE: Bulbostylis juncoides: 303:1-10-81; 407:15-10-81; 424:15-10-81; 606:2910-81; 732:10-11-81; 1045:24-9-82 (B); 1150:4-10-82 (ENCB, MEXU); 3764:31-5-92; Carex marianensis: 822:12-11-81; Cyperus esculentus: 354:8-10-81; 2682:9-7-89; 2723:27-8-89; Cyperus hermaphroditus: 466:18-10-81 (B); 1065:25-9-82; 2677:9-789; Cyperus manimae: 442:18-10-81; 656:2-11-81; 2676:9-7-89; 3361:10-11-90; Cyperus niger: 3842:15-8-92; Cyperus orbicephalus: 2678:9-7-89; Cyperus seslerioides: 144:16-6-81; 2662:8-7-89; 3743:30-5-92; Cyperus spectabilis: 171:19-6-81 (MEXU); 3816:15-8-92; Eleocharis acicularis: 672:3-11-81; 731:10-11-81 (B); 1111:27-9-82 (MEXU); 3761:30-5-92; Eleocharis dombeyana: 3668:17-4-92; 3680:17-4-92; Eleocharis macrostachya: 220:20-6-81 (MEXU); 2683:9-7-89

ERICACEAE: Arbutus glandulosa: 425:15-10-81 (MEXU)

EUPHORBIACEAE: Acalypha indica: 282:30-9-81; 538:21-10-81; 836:12-11-81 (ENCB, MEXU); 1037:24-9-82; Acalypha phleoides: 2681:9-7-89; 3756:30-5-92; Croton dioicus: 3760:30-5-92; Euphorbia hirta: 519:21-10-81; 631:30-10-81 (B, ENCB, MEXU); 1095:27-9-82; 3765:31-5-92; Euphorbia indivisa: 1126:26-9-82; 1148:4-1082; Euphorbia prostrata: 221:20-6-81 (ENCB, MEXU); 309:1-10-81; 551:23-10-81; 666:2-11-81; 945:29-1-82 (B)

FABACEAE: Astragalus micranthus: 226:20-6-81 (MEXU); 1135:27-9-82; 3739:30-5-92; Cologania biloba: 1098:27-9-82; Cologania obovata: 1167:5-10-82; 3752:30-5-92; Dalea foliolosa: 267:26-9-81 (B); 294:1-10-81; 536:21-10-81 (ENCB, MEXU); 690:411-81; Dalea leporina: 315:2-10-81; 278:30-9-81; 1190:6-10-82 (B, ENCB, MEXU); Dalea minutifolia: 293:1-10-81; 399:14-10-81; 854:18-11-81; 874:27-1-82 (B, ENCB, MEXU); 944:29-1-82 (veget.); 952:29-1-82 (veget.); Dalea obovatifolia: 172:19-6-81 (ENCB, MEXU); 418:15-10-81; 535:21-10-81 (B); Dalea reclinata: 433:18-10-81; 673:3-11-81; 1166:5-10-82 (ENCB, MEXU); 1191:6-10-82 (MEXU); Dalea sericea: 142:16-6-81 (ENCB, MEXU); Desmodium grahamii: 125:15-6-81 (B, MEXU); 1099:279-82; Desmodium molliculum: 206:20-6-81 (MEXU); Lupinus leptophyllus: 858:19-1181 (ENCB, MEXU); 862:19-11-81 (B); 3638:4-4-92; Medicago lupulina: 909:28-1-82; 1007:14-3-82; 1134:27-9-82 (ENCB, MEXU); 3678:17-4-92;3749:30-5-92; Medicago polymorpha: 250:18-9-81; 474:18-10-81 (B, MEXU); 929:29-1-82; Mimosa aculeaticarpa: 2663:8-7-89; Phaseolus formosus: 173:19-6-81 (B, ENCB, MEXU); 1129:27-9-82; Pithecellobium leptophyllum: 1011:15-3-82 (B, MEXU); Psoralea rhombifolia: 996:13-3-82; Trifolium amabile: 193:19-6-81 (ENCB, MEXU); 323:2-1081; 589:29-10-81; 698:4-11-81 (B); 1172:5-10-82; Trifolium goniocarpum: 479:18-1081; 539:21-10-81; 588:29-10-81 (B); 640:30-10-81 (ENCB, MEXU); Vicia sativa: 415:15-10-81 (MEXU); Zornia thymifolia: 1023:22-9-82 (MEXU); 3738:30-5-92

FAGACEAE: Quercus castanea: 3650:16-4-92; 3665:17-4-92; Quercus crassipes: 989:232-82; 3636:4-4-92; Quercus aff. laurina: 1152:4-10-92; Quercus mexicana: 995:133-82 (ENCB, MEXU); 1078:26-9-82; 3659:16-4-92; Quercus rugosa: 884:28-1-82 (ENCB, MEXU); 905:28-1-82; 3660:16-4-92

GERANIACEAE: Erodium cicutarium: 343:7-10-81; 382:14-10-81; 486:18-10-81; 717b:9-1181; 775a:11-11-81; Erodium moschatum: 716:9-11-81; 717a:9-11-81; 775b:11-11-81; 
Vibrans: Plantas Vasculares Silvestres en San Juan Quetzalcoapan, Tlaxcala

1025:22-9-82 (ENCB, MEXU); Geranium seemannii: 199:19-6-81; 277:30-9-81; 337:7-10-81; 355:8-10-81; 380:14-10-81 (ENCB, MEXU); 894:28-1-82 (veget.)

HYDROPHYLLACEAE: Nama dichotomum: 253:18-9-81; 279:30-9-81; 1036:24-9-82; 1058:25-9-82

IRIDACEAE: Tigridia meleagris: 2669:9-7-89; Tigridia vanhouttei: 1028:22-9-82 (sólo frutos); 1147:4-10-82 (sólo frutos)

JUNCACEAE: Juncus arcticus: 179:19-6-81; 655:2-11-81 (ENCB, MEXU); 684:4-11-81; 689:4-11-81; 743:10-11-81; 825:12-11-81; 834:12-11-81; Juncus bufonius: 260:259-81; 286:1-10-81; 335:7-10-81; 542:22-10-81; 657:2-11-81 (ENCB, MEXU); 692:411-81; 3667:17-4-92; Juncus microcephalus: 670:2-11-81; 695:4-11-81; 842:18-1181 (ENCB, MEXU); 1017:22-9-82; 2686:9-7-89; 3763:31-5-92; Juncus tenuis: 671:311-81 (ENCB, MEXU); 726:10-11-81; 843:18-11-81

LAMIACEAE: Lepechinia caulescens: 1056:25-9-82 (veget.); 1174:5-10-82; 3823:15-8-92; Marrubium vulgare: 573:23-10-81; 1163:5-10-82 (ENCB, MEXU); Salvia fulgens: 204:20-6-81; Salvia laevis: 2668:9-7-89; Salvia microphylla: 205:20-6-81; 564:23-1081 (ENCB, MEXU); 1101:27-8-82 (cultivado); Salvia polystachya: 168:17-6-81 (ENCB); 445:18-10-81 (MEXU); 524:21-10-81; 1077:26-9-82; 3873:16-8-92; Salvia tiliifolia: 251:18-9-81; Stachys agraria: 198:19-6-81; 595:29-10-81; 823:12-11-81 (ENCB, MEXU); 831:12-11-81

LEMNACEAE: Lemna gibba: 864a:19-11-81; Wolffia columbiana: 864b:19-11-81

LILIACEAE: Calochortus barbatus: 3814:15-8-92; Echeandia flavescens: 133:16-6-81; 164:17-6-81; Echeandia nana: 1125:26-9-82; 3733:30-5-92; Milla biflora: 2658:8-789; Nothoscordum bivalve: 129:15-6-81; 361:8-10-81 (veget.); 744:10-11-81 (veget.)

LOBELIACEAE: Diastatea micrantha: 548:22-10-81 (MEXU); 621:30-10-81; Lobelia fenestralis: 544:22-10-81 (B, MEXU); Lobelia laxiflora: 879:28-1-82 (B); 992:13-3-82; 1003:143-82 (ENCB, MEXU)

LOGANIACEAE: Buddleja cordata: 517:21-10-81 (ENCB, MEXU); 973:29-1-82 (plántulas en un campo de cultivo); 3683:17-4-92; Buddleja perfoliata: 1169:5-10-82 (ENCB, MEXU); Buddleja sessiliflora: 543:22-10-81 (ENCB, MEXU)

MALVACEAE: Kaernemalvastrum lacteum: 579:29-10-81; Kaernemalvastrum subtriflorum: 812:12-11-81; Malva nicaeensis: 1159:5-10-82 (ENCB, MEXU); Malva parviflora: 219:20-6-81; 264:26-9-81; 329:7-10-81; 974:29-1-82; Sphaeralcea angustifolia: 610:29-10-81 (ENCB, MEXU); 3766:31-5-92; Urocarpidium jacens: 287:1-10-81; 288:1-10-81; 577:29-10-81 (ENCB, MEXU); 1200:7-10-82; Urocarpidium limense: 345:7-10-81; 578:29-10-81

NYCTAGINACEAE: Mirabilis jalapa: 478:18-10-81; 1010:15-3-82 (ENCB, MEXU); Mirabilis viscosa: $1100: 27-9-82$

ONAGRACEAE: Gaura hexandra: 545:22-10-81; 675:3-11-81; 3736:30-5-92; Lopezia racemosa: 249:18-9-81; 1024:22-9-82; Oenothera pubescens: 166:17-6-81; 338:710-81; 933:29-1-82 (veget.); Oenothera rosea: 2673:17-7-89; 3674:9-4-92

ORCHIDACEAE: Los especímenes de esta familia fueron determinados por Magdalena Peña y Gerardo Salazar. Brachystele minutiflora: 1001:14-3-82; Corallorrhiza odontorhiza: 1052:25-9-82; Malaxis ehrenbergii: fotografía 5-89; Schiedeella densiflora: 976:20-2-82; Schiedeella aff. eriophora: 977:20-2-82; Schiedeella michuacana: 513:21-10-81, 680:3-11-81; Schiedeella transversalis: 1000:14-3-82 
OXALIDACEAE: Oxalis corniculata: 130:15-6-81 (ENCB, MEXU); 321:2-10-81; 373:14-1081; 405:15-10-81; 469:18-10-81; 914:28-1-82; 934:29-1-82; Oxalis divergens: $128: 15-6-81$; 252:18-9-81 (veget.); 276b:26-9-81; 304:1-10-81; 308:1-10-81; 318:2$10-81 ; 328: 2-10-81 ; 1039: 24-9-82 ; 3735: 30-5-92 ; 3821: 15-8-92 ;$ Oxalis latifolia: 165:17-6-81; 263:25-9-81 (veget.); 265:26-9-81 (veget.); 289:1-10-81; 291:1-10-81; 3737:30-5-92

PAPAVERACEAE: Argemone ochroleuca: (sólo visto); Argemone platyceras: 120:15-6-81; 940:29-1-82

PHYTOLACCACEAE: Phytolacca icosandra: 126:15-6-81; 498:18-10-81

PINACEAE: Los especímenes de Pinus de 1981/82 se perdieron en el correo. Pinus pseudostrobus: $3863: 16-8-92$

PIPERACEAE: Peperomia campylotropa: 1073:25-9-82; 3839:15-8-92

PLANTAGINACEAE: Plantago linearis: 140:16-6-81; 182:19-6-81 (MEXU); 511:19-10-81 (ENCB); 528:21-10-81; Plantago major: 811:12-11-81; 853:18-11-81

POACEAE: Aegopogon cenchroides: 135:16-6-81; 480:18-10-81; 908:28-1-82; 1053:25-982 (CHAP); 1114:26-9-82 (ENCB, MEXU); 3748:30-5-92; Aegopogon tenellus: 485:18-10-81; 592:29-10-81; Agrostis hyemalis: 1090:26-9-82; Aristida appressa: 1115:26-9-82 (CHAP); Aristida divaricata: 419:15-10-81; 530:21-10-81; Aristida laxa: 422:15-10-81; 1120:26-9-82 (CHAP); Aristida schiedeana: 1057:25-9-82 (CHAP); Bothriochloa barbinodis: 852:18-11-81; 1092:26-9-82; 1117:26-9-82; Bothriochloa saccharoides: 851:18-11-81; 1131:27-9-82; Bouteloua hirsuta: 147:16-6-81; 1048:249-82; 3822:15-8-92; Bouteloua scorpioides: 1046:24-9-82 (CHAP); Bouteloua simplex: 302:1-10-81; 638:30-10-81; 639:30-10-81; Brachiaria meziana: 641:30-1081; Brachypodium mexicanum: 375:14-10-81; 903:28-1-82; 1042:24-9-82 (CHAP); 1214:10-10-82; Briza subaristata: 217:20-6-81; 681:4-11-81; 904:28-1-82; 1088:269-82; 2664:8-7-89; Bromus anomalus: 428:15-10-81; 661:2-11-81; 1066:25-9-82 (CHAP); 3869:16-8-92; Bromus carinatus: 151:17-6-81; 180b:19-6-81; 477:18-10-81; 490:18-10-81; 827:12-11-81; 1103:27-9-82 (CHAP); Bromus exaltatus: 3864:16-8-92; Chloris submutica: 192:19-6-81; 339:7-10-81; 408:15-10-81; 416:15-10-81; 462:1810-81; 526:21-10-81; 647:30-10-81; 824:12-11-81; 1067:25-9-82 (CHAP); 1156:5-1082 (ENCB, MEXU); Digitaria leucites: 421:15-10-81; 1061:25-9-82 (CHAP); 1069:259-82; 1187:6-10-82 (CHAP, ENCB, MEXU); 3360:10-11-90; Distichlis spicata: 3769:31-5-92; Echinochloa oplismenoides: 1051:25-9-82 (CHAP, ENCB, MEXU); 3877:16-8-92; Eleusine multiflora: 255:25-9-81; 515:21-10-81; 587:29-10-81; 630:3010-81; 1035:24-9-82; 3770:31-5-92; Eragrostis intermedia: 124:15-6-81; 181:19-6-81; 669:2-11-81; 725:10-11-81; 755:10-11-81;830:12-11-81; 1107:27-9-82 (CHAP); 1151:4-10-82; Eragrostis mexicana: 242:18-9-81; 297:1-10-81; 737:10-11-81; 958:291-82; 1034:24-9-82 (CHAP); 1047:24-9-82; Eragrostis pectinacea: 258:25-9-81; 320:2-10-81; 367:8-10-81; 694:4-11-81; Glyceria fluitans: 1180:6-10-82 (CHAP, ENCB, MEXU); 2689:9-7-8; Hilaria cenchroides: 131:15-6-81; 273:26-9-81; 892:281-82; 1050:24-9-82 (CHAP); 1091:26-9-82; Lolium multiflorum: 3779:31-5-92; Lycurus phleoides: 216:20-6-81; 436:18-10-81; 529:21-10-81; 1049:24-9-82; Muhlenbergia distans: 1146:4-10-82 (MEXU); Muhlenbergia firma: 1165:5-10-82 (CHAP, ENCB, MEXU); Muhlenbergia implicata: 301:1-10-81; 549:22-10-81; 663:211-81; Muhlenbergia macroura: 662:2-11-81; 829:12-11-81; 1082:26-9-82; 1118:26- 
Vibrans: Plantas Vasculares Silvestres en San Juan Quetzalcoapan, Tlaxcala

9-82 (CHAP); Muhlenbergia minutissima: 257:25-9-81; 404: 15-10-81; 738:10-11-81; 1038:24-9-82; Muhlenbergia plumbea: 456:18-10-81; 540:22-10-81; 693:4-11-81; Muhlenbergia pusilla: 327:2-10-81; Muhlenbergia repens: 643:30-10-81; 711a:9-1181; 1128:27-9-82 (CHAP); 3773:31-5-92; Muhlenbergia richardsonis: 1154:4-10-82 (CHAP, ENCB, MEXU); Muhlenbergia rigida: 134:16-6-81; 1068:25-9-82; Muhlenbergia utilis: 3762:30-5-92; Muhlenbergia vaginata: 711b:9-11-81; Panicum bulbosum: 2665:8-7-89; Paspalum distichum: 366:8-10-81 (veget.); 412:15-10-81 (veget.); 1193:7-10-82 (CHAP, ENCB, MEXU; veget.); Pennisetum clandestinum: 3679:17-492; Phalaris canariensis: 763:11-11-81; Piptochaetium fimbriatum: 437:18-10-81; 1055:25-9-82; Poa annua: 359:8-10-81; 451:18-10-81; 603:29-10-81; 718:9-11-81; 3671:17-4-92; Polypogon interruptus: 3670:17-4-92; 3682:17-4-92; Schizachyrium sanguineum: 1116:26-9-82 (CHAP); 2598:29-1-89; 3359:10-11-90; 3831:15-8-92; Setaria geniculata: 668:2-11-81; 2721:27-8-89; Sporobolus indicus: 170:19-6-81; 298:1-10-81; 420:15-10-81; 537:21-10-81; 1040:24-9-82 (CHAP); Stipa ichu: 350:710-81; 417:15-10-81; 448:18-10-81; 520: 21-10-81; 900:28-1-82; 1140:26-9-82; Stipa mucronata: 180a:19-6-81; 376:14-10-81; 476:18-10-81; 833:12-11-81; 1041:24-9-82 (CHAP); Stipa tenuissima: 210:20-6-81; 1145:4-10-82 (ENCB, MEXU); 3750:30-5-92; Trisetum deyeuxioides: 703:4-11-81; 1086:26-9-82 (CHAP); 1094:27-9-82; Trisetum kochianum: 441:18-10-81; 727:10-11-81; 1173:5-10-82 (CHAP); Vulpia myuros: 440:18-10-81; 481:18-10-81; 488:18-10-81; 917:28-1-82; 956:29-1-82; 1084:26-9-82; $1102: 27-9-82 ; 1138: 27-9-82$

POLEMONIACEAE: Loeselia mexicana: 396:14-10-81 (ENCB, MEXU); 3673:17-4-92

POLYGONACEAE: Polygonum aviculare: 197:19-6-81; 356:8-10-81; 746:10-11-81; Polygonum mexicanum: 844:18-11-81 (ENCB, MEXU); 868:17-12-81; 2684:9-7-89; Rumex crispus: 3817:15-8-92; Rumex mexicanus: 191:19-6-81; 310:1-10-81; 598:29-10-81; 658:2-11-81; 667:2-11-81; 742:10-11-81; 780:11-11-81 (veg.); 902:28-1-82 (veg.); Rumex obtusifolius: 581:29-10-81; 899:28-1-82; 1087:26-9-82

PORTULACACEAE: Calandrinia micrantha: 409:15-10-81; 730:10-11-81; 848:18-11-81 (ENCB, MEXU); 1106:27-9-82; Portulaca oleracea: 796:11-11-81

PRIMULACEAE: Anagallis arvensis: 2666:9-7-89

RESEDACEAE: Reseda luteola: 324:2-10-81; 340:7-10-81; 402:15-10-81; 927:29-1-82 (MEXU)

RHAMNACEAE: Adolphia infesta: 3677:17-4-92

ROSACEAE: Alchemilla aphanoides: 377:14-10-81; 390:14-10-81; Amelanchier denticulata: 3652:16-4-92; Crataegus pubescens: 518: 21-10-81; 863:19-11-81; 893:28-1-82 (ENCB, MEXU); Potentilla sp: 3841:15-8-92 (veget.); Prunus microphylla: 2670:9-789; Prunus serotina: 869:17-12-81 (ENCB, MEXU); Rubus liebmannii: 987:23-2-82

RUBIACEAE: Bouvardia ternifolia: 121:15-6-81; 771:11-11-81; Crusea diversifolia: 1026:229-82; Galium aschenbornii: 845:18-11-81; 1215:10-10-82; Galium uncinulatum: 819:12-11-81 (ENCB, MEXU); 906:28-1-82; 911:28-1-82; Relbunium microphyllum: 213:20-6-81; 403:15-10-81 (ENCB, MEXU); 1121:26-9-82; 1132:27-9-82; 2661:8-789; Richardia tricocca: 143:16-6-81; 532:21-10-81

SCROPHULARIACEAE: Bacopa procumbens: 541:22-10-81; 654:2-11-81; 1096:27-9-82; Castilleja arvensis: 266:26-9-81; 306:1-10-81; 950:29-1-82; 971:29-1-82; Castilleja lithospermoides: 203:20-6-81; Castilleja tenuiflora: 137:16-6-81 (ENCB, MEXU); 
449:18-10-81; 898:28-1-82; 1018:22-9-82; Lamourouxia dasyantha: 388:14-10-81 (ENCB, MEXU); 1218:10-10-82;; Lamourouxia multifida: 386:14-10-81; 1020:22-9-82; Limosella aquatica: 262:25-9-81; Linaria canadensis: 316:2-10-81 (veget.); 953:291-82; 1012:15-3-82 (ENCB, MEXU); 3358:10-11-90; Penstemon barbatus: 3759:305-92; Penstemon roseus: 188:19-6-81 (ENCB, MEXU); 508:19-10-81; Verbascum virgatum: 722:10-11-81; Veronica peregrina: 259:25-9-81; 347:7-10-81

SOLANACEAE: Datura stramonium: 154:17-6-81; 648:30-10-81 (ENCB, MEXU); 779:11-1181; Jaltomata procumbens: 225:20-6-81; 344b:7-10-81 (veget.); 357:8-10-81; 414:1510-81; 497b:18-10-81; 799:11-11-81; 2679:9-7-89; Physalis chenopodiifolia: 190:196-81; 493:18-10-81 (MEXU); 582:29-10-81 (ENCB); Physalis foetens: 184:19-6-81 (ENCB, MEXU); 720:9-11-81; Physalis philadelphica: 770:11-11-81; 1137:27-9-82; Solanum demissum: 183:19-6-81; Solanum lanceolatum: 877:28-1-82 (ENCB, MEXU); 979:22-2-82; Solanum nigrescens: 175:19-6-81; 379:14-10-81 (MEXU, ENCB); 497a:18-10-81; 768:11-11-81; Solanum rostratum: 593:29-10-81; 607:29-1081; Solanum stoloniferum: 3776:31-5-92

URTICACEAE: Parietaria pensylvanica: 762:11-11-81; Urtica subincisa: 468:18-10-81; 704, 705, 706:9-11-81 (ENCB, MEXU); Urtica urens: 604:29-10-81; 774:11-11-81

VALERIANACEAE: Valeriana sorbifolia: 1019:22-9-82

VERBENACEAE: Verbena bipinnatifida: 167:17-6-81 (ENCB, MEXU); 292:1-10-81; 374:1410-81; Verbena carolina: 169:17-6-81; 378:14-10-81; 583:29-10-81; 875:28-1-82; 1195:7-10-82 (ENCB, MEXU); 3363:10-11-90;3753:30-5-92;3771a:31-5-92; Verbena menthaefolia: 741:10-11-81; 1196:7-10-82; 3771b:31-5-92; Verbena teucriifolia: 928:29-1-82 
Apéndice 3. Nombres comunes enumerados por orden alfabético con sus nombres científicos correspondientes.

abrojo
acacia
acahual
aceitilla
ajonjolillo
alfalfilla
alfilerillo
alfombrilla
alita de angel
alpiste
amamaxctle
anisillo
anisillo amarillo
árnica
asomiate (del campo)
asomiate del monte
atenclaco
barba de chivo
barrequedito
bejuquillo
bolsa del pastor
bretónica
cabezona
canelillo
capulín
cebentón
cebolleta
cenicilla
cenicilla blanca
cenicillo
chía
chicalote
chicalote amarillo
chichicamol
chichicaxtle
chilacoco
chilillo
chincuento
chingüento
chinguiñosa
chipule

Adolphia infesta

Dalea minutifolia

Simsia amplexicaulis

Bidens odorata

Lepidium virginicum, L. oblongum, L. schaffneri

Polygonum aviculare

Erodium cicutarium, E. moschatum

Verbena bipinnatifida, $V$. teucriifolia

Eupatorium petiolare

Phalaris canariensis

Rumex mexicanus

Tagetes micrantha

Schkuhria pinnata

Heterotheca inuloides

Senecio salignus

Verbesina virgata

Baccharis salicifolia

Stipa ichu

Dalea minutifolia

Ipomoea purpurea

Capsella bursa-pastoris

Lepechinia caulescens

Gomphrena pringlei

Conyza coronopifolia, Gnaphalium stramineum

Prunus serotina ssp. capuli

Phytolacca icosandra

Nothoscordum bivalve

Conyza filaginoides

Conyza coronopifolia

Helianthemum glomeratum

Salvia polystachya, S. tiliifolia

Argemone platyceras

Argemone ochroleuca

Microsechium helleri

Urtica subincisa, U. urens

Prionosciadium thapsoides

Lobelia laxiflora

Florestina pedata

Gaura hexandra

Conyza canadensis

Pinaropappus roseus, Taraxacum officinale 


chisme blanco
chucuyul
cilantrillo
cimonilla
ciridoña
ciridoña morada (roja)
clalayote
claldurazno
clalesquite
clalocote
clalpipitza
clavelillo
cola de diablo
cola de zorra
colchoncillo
ctlalesquite
ctlamacas
ctlapalasol
diente de león
doradilla
duraznillo
ebol
encino
engordacabras
epazote (morado)
epazote de zorrillo
escobilla blanca,
escorzonera
estafiate
estrellita

flor de muerto

frijolillo gigantón girasol gobernadora gobernadora morada gordolobo guistomate heno hierba ceniza hierba de coyote hierba de golpe
Sedum bourgaei

Oxalis divergens, O. latifolia

Adiantum poiretii

Conyza sophiifolia

Euphorbia prostrata

Euphorbia hirta var. procumbens

Matelea sp. (decumbens o prostrata)

Amelanchier denticulata

Cyperus spectabilis

Baccharis pteronioides, Haplopappus venetus

Kuhnia rosmarinifolia

Pinaropappus roseus, Saponaria officinalis

Metastelma angustifolium

Reseda luteola

Pennisetum clandestinum

Cyperus esculentus, C. hermaphroditus, C. manimae Stevia subpubescens

Sicyos deppei

Taraxacum officinale

Selaginella rupestris

Solanum rostratum

Vicia sativa

Quercus castanea, Q. crassipes, Q. laurina, Q. mexicana

Dalea minutifolia

Chenopodium ambrosioides

Chenopodium graveolens

Baccharis pteronioides

Eryngium carlinae

Artemisia ludoviciana ssp. mexicana

Arenaria lanuginosa, A. lycopodioides, Galinsoga parviflora, G. quadriradiata, Milla biflora, Richardia tricocca ssp. tetracocca

Tagetes erecta

Phaseolus formosus

Simsia amplexicaulis

Dahlia coccinea, Cosmos bipinnatus

Stevia nepetifolia

Stevia pilosa, S. viscida

Gnaphalium oxyphyllum

Solanum lanceolatum

Tillandsia usneoides, $T$. recurvata

Eupatorium glabratum

Castilleja arvensis

Baccharis salicifolia 
Vibrans: Plantas Vasculares Silvestres en San Juan Quetzalcoapan, Tlaxcala

hierba de hinchazón

hierba de jiondra

hierba de la víbora

hierba de la virgen

hierba de mosco

hierba de pollo

hierba de ratón

hierba de San Nicolás

hierba de sombra

hierba de zopilote

hierba del cáncer

hierba del grano

hierba del negro

hierba del pastor

hierba mora

hocico de coyote

ixtecuate

jaboncillo

jaltomate

jaramao, jaramado

jarilla

jarrito

jarrito de campo

jícama

jicamita cimarrona

jojoxchichil

lanté(n)

lechuga

lechuguilla

lengua de pájaro

lengua de vaca

lentejilla

linda tarde

mactlalillo

madroño

malva

malva china

manto

maravilla

maravillita

marrubio

mazorquilla

metatera

metezurras

mirasol
Alchemilla aphanoides

Datura stramonium

Castilleja tenuiflora

Loeselia mexicana

Reseda luteola

Tinantia erecta, Tripogandra purpurascens

Dalea leporina, D. sericea

Piqueria trinervia

Arenaria lanuginosa

Physalis foetens

Acalypha indica

Stachys agraria

Sphaeralcea angustifolia

Plantago linearis

Solanum nigrescens

Castilleja arvensis, C. lithospermoides

Solanum rostratum

Saponaria officinalis

Physalis chenopodiifolia

Raphanus raphanistrum

Eupatorium glabratum

Lamourouxia multifida, Penstemon roseus

Lamourouxia dasyantha

Oxalis divergens, O. latifolia

Psoralea rhombifolia

Eupatorium petiolare

Plantago major

Sonchus oleraceus

Hieracium schultzii, Sonchus oleraceus

Calandrinia micrantha

Buddleja sessiliflora, Rumex mexicanus, R. obtusifolius

Lemna gibba, Wolffia columbiana

Oenothera pubescens

Tripogandra purpurascens

Arbutus glandulosa

Malva nicaeensis, M. parviflora

Urocarpidium jacens, U. limense

Ipomoea purpurea

Mirabilis jalapa

Mirabilis viscosa

Marrubium vulgare

Lupinus leptophyllus

Silene laciniata

Aster subulatus

Cosmos bipinnatus 
mirto

mirto grande

mora

mozoquelite

mozoquelite amarillo

mozoquelitillo

nabillo

nabo

navajilla

nexguili

nopal

nopal de la ardilla

ocote

ojito de gallo

ojo de gallo

oreja de burro

ortiga

paletaria, paletillo

palma

palmillo

palmita

papa cimarrona

pastito de humedad

pasto de agua

pasto de grama

pasto de humedad

pata de león

paxtle

pegarropa

pegatrapo

perillo

perlilla

pextotl

pextotl chico

picos del rey

pimentillo

pino

pioja

piojita

pipisco

pipitza cimarrona

plumajillo

púlida
Salvia microphylla

Salvia fulgens

Solanum nigrescens

Bidens odorata

Achyropappus anthemoides, Bahia xylopoda, Bidens anthemoides

Sabazia humilis

Descurainia impatiens, Rorippa mexicana

Brassica rapa

Bouteloua hirsuta, B. scorpiodes, B. simplex

Chenopodium murale

Opuntia robusta var. robusta, O. velutina

Opuntia robusta var. larreyi

Pinus leiophylla, P. montezumae, P. pseudostrobus

Commelina tuberosa

Sanvitalia procumbens

Echeveria gibbiflora, E. mucronata, E. subrigida

Urtica subincisa

Parietaria pensylvanica

Brachystele minutiflora, Schiedeella densiflora, $S$. eriophora

Notholaena aurea

Echeandia flavescens

Solanum demissum

Juncus bufonius

Paspalum distichum

Chloris submutica

Paspalum distichum, Polypogon interruptus

Geranium seemannii

Tillandsia usneoides, $T$. recurvata

Desmodium grahamii

Desmodium molliculum

Lopezia racemosa

Symphoricarpos microphyllus

Brickellia secundiflora

Brickellia veronicifolia

Juncus arcticus var. mexicanus

Peperomia campylotropa

Cupressus lindleyi

Eragrostis mexicana

Muhlenbergia minutissima

Jaltomata procumbens

Kuhnia rosmarinifolia

Achillea millefolium

Drymaria villosa, D. malachioides, D. glandulosa 
Vibrans: Plantas Vasculares Silvestres en San Juan Quetzalcoapan, Tlaxcala

púlida grande

púlida hembra

púlida macho

quelite

queltonil

quiebraplatos

rabo de puerco

ratoncillo

reventón

romerillo

sabino

sacahul

salverreal de bolita

siempreviva

sinvergüenza

té negro

tecayatillo

tecayatito (del campo)

tejocote

tepopote

tepozán

tesguate

tianquis pepetla

tomate

tonta chuate, tonta juate

trébol

trebolillo

trompetilla

tul

tzompanctle

uña de gato

vara de cohete

vara de San Joaquín

verbena

verdolaga

violetilla

yepaclina

zarzamora

zoapaxtle
Arenaria lanuginosa

Arenaria lanuginosa, Drymaria glandulosa

Oxalis corniculata

Chenopodium berlandieri

Amaranthus hybridus

Zephyranthes brevipes

Cologania biloba, C. obovata

Dalea foliolosa, $D$. obovatifolia, $D$. sericea

Phytolacca icosandra

Asclepias linaria

Juniperus deppeana

Sporobolus indicus

Buddleja perfoliata

Sedum praealtum ssp. parvifolium

Oenothera rosea

Bidens aurea

Dyssodia papposa

Tagetes lunulata

Crataegus pubescens

Baccharis conferta

Buddleja cordata

Quercus rugosa

Alternanthera caracasana, Guilleminea densa

Physalis philadelphica

Datura stramonium

Medicago polymorpha

Trifolium amabile, T. goniocarpum

Bouvardia ternifolia

Eleocharis macrostachya, Juncus arcticus var. mexi-

canus, J. microcephalus, J. tenuis var. dichotomus

Buddleja cordata

Mimosa aculeaticarpa, Pithecellobium leptophyllum

Baccharis salicifolia

Verbascum virgatum

Verbena carolina, V. mentaefolia

Portulaca oleracea

Bacopa procumbens

Chenopodium graveolens

Rubus liebmannii

Montanoa tomentosa 